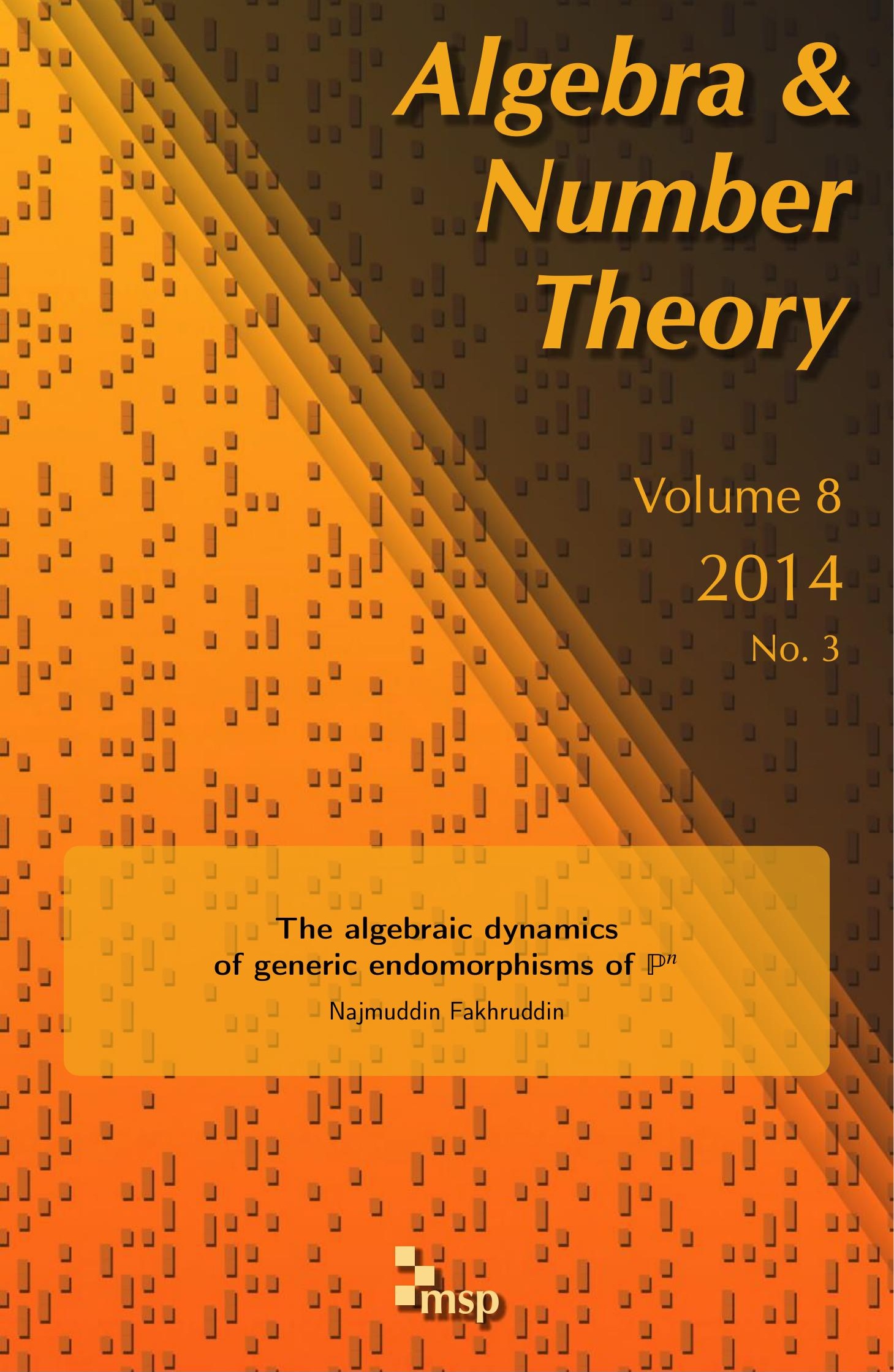




\title{
The algebraic dynamics of generic endomorphisms of $\mathbb{P}^{n}$
}

\author{
Najmuddin Fakhruddin
}

\begin{abstract}
We investigate some general questions in algebraic dynamics in the case of generic endomorphisms of projective spaces over a field of characteristic zero. The main results that we prove are that a generic endomorphism has no nontrivial preperiodic subvarieties, any infinite set of preperiodic points is Zariski-dense and any infinite subset of a single orbit is also Zariski-dense, thereby verifying the dynamical "Manin-Mumford" conjecture of Zhang and the dynamical "MordellLang" conjecture of Denis and Ghioca and Tucker in this case.
\end{abstract}

\section{Introduction}

The goal of this article is to study some aspects of the algebraic dynamics of generic endomorphisms ${ }^{1}$ of $\mathbb{P}^{n}$ of degree $d>1$ over a field $K$ of characteristic zero. Properties of algebraic varieties, for example smooth projective curves or abelian varieties, are often easier to derive for generic varieties than for arbitrary varieties, the main reason being that one has a great deal of freedom in choosing specialisations. It is natural to expect that the same holds for algebraic dynamical systems; we show that this is indeed the case for generic endomorphisms of $\mathbb{P}^{n}$. We prove three results for such endomorphisms: two of them have analogues expected to hold much more generally, though at present this is far from being known.

Our main result is:

Theorem 1.1. Let $f: \mathbb{P}_{K}^{n} \rightarrow \mathbb{P}_{K}^{n}$ be a generic endomorphism of degree d $>1$ over an algebraically closed field $K$ of characteristic zero. For each $x \in \mathbb{P}^{n}(K)$, every infinite subset of $O_{f}(x)$, the $f$-orbit of $x$, is Zariski-dense in $\mathbb{P}_{K}^{n}$.

This implies the dynamical "Mordell-Lang" conjecture of [Denis 1994; Ghioca and Tucker 2009] for generic endomorphisms. This conjecture has been proved for étale endomorphisms of arbitrary varieties by Bell, Ghioca and Tucker [Bell et al.

MSC2010: primary 37P55; secondary 37F10.

Keywords: generic endomorphisms, projective space.

${ }^{1}$ The precise meaning of generic endomorphism is given in Definition 3.1, but we note here that when $K=\mathbb{C}$ this means we consider endomorphisms in the complement of a countable union of proper subvarieties of the natural parameter variety of endomorphisms of degree $d$. 
2010], but there are only a few other cases where it is known. The proof of this theorem is based on two other results. The first is:

Theorem 1.2. Let $f: \mathbb{P}_{K}^{n} \rightarrow \mathbb{P}_{K}^{n}$ be a generic endomorphism of degree $d>1$ over an algebraically closed field $K$ of characteristic zero. If $X \subset \mathbb{P}_{K}^{n}$ is an irreducible subvariety such that $f^{r}(X)=X$ for some $r>0$, then $X$ is a point or $X=\mathbb{P}_{K}^{n}$.

This is a rather straightforward consequence of the transitivity of the monodromy action on the set of periodic points of a fixed period of a generic endomorphism, which we prove (Proposition 3.3) using a result of Bousch [1992], Lau and Schleicher [1994] and Morton [1998] for polynomials in one variable of the form $z \mapsto z^{d}+c$.

We then extend Proposition 3.3 to prove transitivity of the monodromy action on the set of preperiodic points of fixed period and preperiod of a generic endomorphism (Proposition 4.6); this does not hold for the 1-parameter family of polynomials mentioned above, and we use a 2-parameter family containing this. This allows us to prove Zhang's "Manin-Mumford" conjecture [Ghioca et al. 2011] in the case of generic endomorphisms in the following strong form:

Theorem 1.3. For $f: \mathbb{P}_{K}^{n} \rightarrow \mathbb{P}_{K}^{n}$ a generic endomorphism of degree $d>1$ over an algebraically closed field $K$ of characteristic zero, any infinite subset of $\mathbb{P}^{n}(K)$ consisting of $f$-preperiodic points is Zariski-dense in $\mathbb{P}_{K}^{n}$.

We prove Theorem 1.1 by combining Theorems 1.2 and 1.3 with some $p$-adic as well as mod $p$ arguments. Note that the statement does not involve (pre)periodic points in any way. However, using a lifting argument for periodic points, we show that any subvariety $Y$ containing an infinite subset of $O_{f}(x)$ must contain infinitely many periodic points, or $x$ can be specialised in such a way that one may apply the $p$-adic interpolation argument used in [Bell et al. 2010] to prove the conjecture for étale endomorphisms. Theorems 1.3 and 1.2 then force $Y$ to be equal to $\mathbb{P}_{K}^{n}$ in either of these cases.

\section{Preliminaries}

Let $X$ be a set and $f: X \rightarrow X$ any map. By $f^{n}$, we shall mean the $n$-fold composite of $f$ with itself. For $x \in X$, we denote by $O_{f}(x)$ its orbit under $f$, i.e., the set $\left\{f^{n}(x)\right\}_{n \geq 0}$. A point $x \in X$ is said to be $f$-periodic if $f^{n}(x)=x$ for some $n>0$. The smallest such integer is called the period of $x$. We denote the set of all periodic points of period $b$ by $\operatorname{Per}_{f}(b)$. A point $x \in X$ is said to $f$-preperiodic if $O_{f}(x)$ is finite. The preperiod of $f$ is the smallest nonnegative integer $a$ such that $f^{a}(x)$ is periodic, and the period of $x$ is the period of any periodic point in its orbit. We denote by $\operatorname{Prep}_{f}(a, b)$ the set of all such points. Let $\operatorname{Orb}_{f}(b)$ denote the set of orbits of $f$-periodic points of period $b$. If this is finite, then $\left|\operatorname{Per}_{f}(b)\right|=b \cdot\left|\operatorname{Orb}_{f}(b)\right|$. We drop $f$ from any of the notation introduced above if there is no scope for confusion. 
If $X$ is an algebraic variety over a field $K$ and $f: X \rightarrow X$ is a morphism over $K$, we use the same notation as above for the induced map on the set of $L$-rational points of $X$ for any extension field $L$ of $K$.

Let $S$ be a smooth irreducible variety over a field $k$, and let $g: Z \rightarrow S$ be a finite flat morphism. By the monodromy or Galois action of $g$, we shall mean the action of $\operatorname{Gal}(\overline{k(S)} / k(S))$ on (the points of) a geometric generic fibre of $g$. If $g$ is generically smooth - this is always true if $\operatorname{char}(k)=0$ and $Z$ is reduced - there is a Zariski open subset $U$ of $S$ such that $g$ induces a finite étale morphism $g^{-1}(U) \rightarrow U$, and then the monodromy may be interpreted as an action of $\pi_{1}^{\text {et }}(U, *)$, where $*$ is a geometric point of $U$. If $k=\mathbb{C}$, it may be interpreted as an action of $\pi_{1}^{\text {top }}(U, *)$.

The monodromy action is transitive if and only if $Z$ is irreducible. If $Z$ is generically smooth, this is equivalent to $Z^{0}$, the smooth locus of $Z$, being connected or, if $k=\mathbb{C}$, path-connected.

Definition 2.1. Let $\pi: \mathscr{L} \rightarrow S$ be a projective morphism and $f: \mathscr{X} \rightarrow \mathscr{X}$ a surjective morphism over $S$. We say that $f$ is quasipolarised if there exists a line bundle $\mathscr{L}$ on $\mathscr{X}$ such that $f^{*}(\mathscr{L}) \otimes \mathscr{L}^{-1}$ is $S$-ample.

For any morphism $\pi: \mathscr{L} \rightarrow S$ and $f: \mathscr{L} \rightarrow \mathscr{L}$ a morphism over $S$, we denote by $\Gamma_{f}$ the graph of $f$ in $\mathscr{X} \times_{S} \mathscr{X}$. Let $P_{f}(n)$ be the closed subscheme of $\mathscr{X}$ defined by the intersection of $\Gamma_{f^{n}}$ with the diagonal. A geometric point of the fibre of $P_{f}(n)$ over any point $s \in S$ is a periodic point of period dividing $n$ of the map $f_{s}$ of $\mathscr{L}_{s}$ induced by $f$. Similarly, let $P_{f}(m, n)$ be the intersection of $\Gamma_{f^{m}}$ and $\Gamma_{f^{n}}$, which we view as a subscheme of $\mathscr{X}$ via the first projection.

Lemma 2.2. Let $\pi: \mathscr{X} \rightarrow S$ be a smooth projective morphism with $S$ a regular irreducible finite-dimensional scheme, and let $f: \mathscr{X} \rightarrow \mathscr{X}$ be a finite quasipolarised morphism. Then:

(1) For any $m, n \geq 0, m \neq n, P_{f}(m, n)$ is finite and flat over $S$.

(2) For any $s_{1}$ and $s_{2}$ in $S$ with $s_{2}$ a specialisation of $s_{1}$, any element of $\operatorname{Prep}_{f_{s_{2}}}(a, b)$ can be lifted to an element of $\operatorname{Prep}_{f_{s_{1}}}(a, b)$.

Proof. Since $f$ is proper, $P_{f}(m, n)$, being a closed subscheme of $\mathscr{X}$, is also proper over $S$. The dimension of each irreducible component of $P_{f}(m, n)$ is at least equal to $\operatorname{dim}(S)$ since the codimension of $\Gamma_{f^{i}}$ in $\mathscr{X} \times_{S} \mathscr{X}$ is the relative dimension of $\mathscr{X}$ over $S$. To prove $P_{f}(m, n)$ is finite over $S$, it suffices to show that the fibres of this map are finite since proper quasifinite morphisms are finite. Furthermore, the finiteness of the fibres implies that the dimension of each component of $P_{f}(m, n)$ is exactly $\operatorname{dim}(S)$; hence, $P_{f}(m, n)$ is a local complete intersection in $\mathscr{X} \times_{S} \mathscr{X}$ that is regular, which implies that $P_{f}(m, n)$ is Cohen-Macaulay. Since the dimension of each irreducible component of $P_{f}(m, n)$ is at least $\operatorname{dim}(S)$, each such component 
dominates $S$ if all the fibres are finite. It then follows from the fibrewise flatness criterion that all of (1) is a consequence of the finiteness of the fibres.

Let $\mathscr{L}$ be a line bundle on $\mathscr{X}$ so that $M=f^{*}(\mathscr{L}) \otimes \mathscr{L}^{-1}$ is ample. Then $\left(f^{m}\right)^{*}(\mathscr{L})=$ $\mathscr{L} \otimes M \otimes f^{*}(M) \otimes \cdots \otimes\left(f^{m-1}\right)^{*}(M)$ and similarly for $n$. By the construction of $P_{f}(m, n),\left(f^{m}\right)^{*}(\mathscr{L})$ and $\left(f^{m}\right)^{*}(\mathscr{L})$ restrict to the same line bundle on it, so assuming $m>n$ without loss of generality, we get that $\left(f^{m-1}\right)^{*}(M) \otimes \cdots \otimes$ $\left(f^{n}\right)^{*}(M)$ is trivial on $P_{f}(m, n)$. But $M$ is ample; hence, so is $\left(f^{i}\right)^{*}(M)$ for all $i \geq 0$ since $f$ is finite. There is at least one factor in the above tensor product of line bundles, so this is only possible if all the fibres are finite.

If $x \in \operatorname{Prep}_{f_{s_{2}}}(a, b)$, then $x$ occurs in the fibre of $P_{f}(a+b, a)$ over $s_{1}$ and does not occur in the fibre of any $P_{f}(m, n)$ for $m<a+b$ or $n<a$. By (1), there is a point $\tilde{x}$ in the fibre of $P_{f}(a+b, a)$ specialising to $x$. By the definition of $P_{f}(m, n)$, it follows that $f_{s_{1}}^{a+b}(\tilde{x})=f_{s_{1}}^{a}(\tilde{x})$, so $\tilde{x}$ is preperiodic with preperiod $\leq a$ and period $\leq b$. Since neither the preperiod nor the period can increase under specialisation and $\tilde{x}$ specialises to $x$, (2) follows.

\section{Periodic points and periodic subvarieties}

Let Mor $_{n, d}$ be the scheme over $\mathbb{Z}$ representing morphisms of $\mathbb{P}_{\mathbb{Z}}^{n}$ to itself of algebraic degree $d$. Its $k$-valued points, for any field $k$, consist of $(n+1)$-tuples of homogeneous polynomials of degree $d$ over $k$ without common zeros in $\mathbb{P}_{k}^{n}$ up to a scalar. It is smooth and of finite type over $\mathbb{Z}$ and has geometrically irreducible fibres. For any field $L$, we denote $\operatorname{Mor}_{n, d} \times \operatorname{Spec}(\mathbb{Z}) \operatorname{Spec}(L)$ by $\operatorname{Mor}_{n, d / L}$.

Definition 3.1. If $K$ is an algebraically closed field, we say that an endomorphism $f$ of $\mathbb{P}_{K}^{n}$ is generic if the image of the induced map $\operatorname{Spec}(K) \rightarrow \operatorname{Mor}_{n, d}$ corresponding to some conjugate of $f$ by an element of $\operatorname{PGL}_{n+1}(K)=\operatorname{Aut}\left(\mathbb{P}_{K}^{n}\right)$ is the generic point of a fibre of the structure morphism $\operatorname{Mor}_{n, d} \rightarrow \operatorname{Spec}(\mathbb{Z})$.

If $K=\mathbb{C}$, the set of points in $\operatorname{Mor}_{n, d}(\mathbb{C})$ corresponding to generic morphisms is the complement of a countable union of proper subvarieties.

We recall the theorem of Bousch [1992, Chapitre 3, Théorème 4], Lau and Schleicher [1994, Theorem 4.1] and Morton [1998, Theorem D] alluded to earlier; the statement below is [Morton 1998, Theorem 10] except that we have replaced the field $\overline{\mathbb{Q}}$ there by $k$.

Theorem 3.2. Let $k$ be a field of characteristic zero, and let $f(z)=z^{d}+t$ with $t$ transcendental over $k$ and $d \geq 2$. For any $b \geq 1$, the Galois group of the polynomial $f^{b}(z)-z$ over $k(t)$ is the direct product $\prod_{e \mid b}\left(\mathbb{Z} / e \mathbb{Z}\right.$ wr $\left.S_{r_{e}}\right)$, where wr denotes the wreath product and $e \cdot r_{e}$ is the number of periodic points of period e over $\overline{k(t)}$.

The theorem can be interpreted as saying that the Galois action is as large as possible given that it must commute with the action of $f$. One may expect that a 
similar result holds for generic endomorphisms of projective spaces of arbitrary dimension; however, the following proposition, for the proof of which we will use only the transitivity of the Galois action in the above theorem, suffices for our applications:

Proposition 3.3. Let $k$ be a field of characteristic zero, and let $k_{n, d}$ be the function field of Mor $_{n, d / k}$. Let $f_{n, d}$ be the endomorphism of $\mathbb{P}_{k_{n, d}}^{n}$ corresponding to the generic point of $\operatorname{Mor}_{n, d / k}$, and let $b$ be any positive integer. Then $\operatorname{Gal}\left(\overline{k_{n, d}} / k_{n, d}\right)$ acts transitively on $\operatorname{Per}_{f_{n, d}}(b)$.

For a field $k$ and any element $\lambda \in k$, let $\phi_{\lambda}: \mathbb{A}_{k}^{1} \rightarrow \mathbb{A}_{k}^{1}$ be the map given by $z \mapsto z^{d}+\lambda$; the integer $d$ will be assumed to be fixed whenever we use this notation. The periodic points of $\phi_{0}$ are 0 and the roots of unity of order prime to $d$ : if $\zeta$ is a primitive $n$-th root of unity with $(n, d)=1$, then the period of $\zeta$ is the order of $d$ in $(\mathbb{Z} / n \mathbb{Z})^{\times}$.

We shall need the following simple lemma for the proof of Proposition 3.3:

Lemma 3.4. Let $d>1$ and $m, m^{\prime} \geq 1$ be integers such that $(m, d)=\left(m^{\prime}, d\right)=1$. Assume that the highest powers of 2 dividing $m$ and $m^{\prime}$ are unequal or are both equal to 1 . Let $a$ and $a^{\prime}$ be the orders of $d$ in $(\mathbb{Z} / m \mathbb{Z})^{\times}$and $\left(\mathbb{Z} / m^{\prime} \mathbb{Z}\right)^{\times}$, respectively.

(1) The order of $d$ in $\left(\mathbb{Z} / \operatorname{lcm}\left(m, m^{\prime}\right) \mathbb{Z}\right)^{\times}$is divisible by $\operatorname{lcm}\left(a, a^{\prime}\right)$.

(2) There exist roots of unity $\zeta$ and $\zeta^{\prime}$ of orders $m$ and $m^{\prime}$, respectively, so that $\zeta \zeta^{\prime-1}$ is of order $\operatorname{lcm}\left(m, m^{\prime}\right)$.

(3) For $\zeta$ and $\zeta^{\prime}$ as above, there exists a primitive $\operatorname{lcm}\left(m, m^{\prime}\right)$-th root of unity $\eta$ so that $\eta \zeta$ is a primitive $\operatorname{lcm}\left(m, m^{\prime}\right)$-th root of unity.

Proof. The natural quotient maps from $\mathbb{Z} / \operatorname{lcm}\left(m, m^{\prime}\right) \mathbb{Z}$ to $\mathbb{Z} / m \mathbb{Z}$ and $\mathbb{Z} / m^{\prime} \mathbb{Z}$ induce group homomorphisms from $\left(\mathbb{Z} / \operatorname{lcm}\left(m, m^{\prime}\right) \mathbb{Z}\right)^{\times}$to $(\mathbb{Z} / m \mathbb{Z})^{\times}$and $\left(\mathbb{Z} / m^{\prime} \mathbb{Z}\right)^{\times}$, respectively. This implies that the order of $d$ in $\left(\mathbb{Z} / \operatorname{lcm}\left(m, m^{\prime}\right) \mathbb{Z}\right)^{\times}$is divisible by $\operatorname{lcm}\left(a, a^{\prime}\right)$.

To prove (2) and (3), we may reduce to the case that $m$ and $m^{\prime}$ are powers of the same prime $p$. Let $P \subset \mathbb{Z} / p^{r} \mathbb{Z}$ be the set of generators, so $|P|=p^{r}-p^{r-1}$. If $p>2$, then $|P|>p^{r-1}$, so the translate of $P$ by any element of $\mathbb{Z} / p^{r} \mathbb{Z}$ has a nonempty intersection with $P$. If $p=2$, the claim follows from the extra condition since the translate of $P$ by an element not in $P$ always intersects $P$ nontrivially.

Proof of Proposition 3.3. If $n=1$ and $b>1$, the proposition follows immediately from Theorem 3.2. If $b=1$, a much simpler version of the argument below shows transitivity; since we do not use this later, we leave the details to the reader.

We now assume $n>1$. Consider the morphism $g_{n, d}: \mathbb{P}_{k}^{n} \rightarrow \mathbb{P}_{k}^{n}$ given by $\left[x_{0}, \ldots, x_{n}\right] \mapsto\left[x_{0}^{d}, \ldots, x_{n}^{d}\right]$. The set $\operatorname{Per}_{g_{n, d}}(b)$ consists of points that have a representative $\left[\xi_{0}, \xi_{1}, \ldots, \xi_{n}\right]$ with each $\xi_{i}$ equal to 0 or a $\left(d^{b}-1\right)$-th root of unity. 
The standard affine charts of $\mathbb{P}_{k}^{n}$ given by the locus where a fixed coordinate is nonzero are preserved by $g_{n, d}$. A simple computation on each such chart shows that the eigenvalues of the differential of $g_{n, d}^{b}$ at a fixed point are equal to $d^{b} \xi$, where $\xi$ is a root of unity or 0 . This is never equal to 1 since $d>1$, so $\Gamma_{g_{n, d}^{b}}$ and the diagonal intersect transversely in $\mathbb{P}_{k}^{n} \times \mathbb{P}_{k}^{n}$ for all $b>0$. Consequently, all periodic points of $g_{n, d}$ have multiplicity 1, so we may use $g_{n, d}$ as a basepoint in Mor ${ }_{n, d}$ in order to compute the Galois action on $\operatorname{Per}_{f_{n, d}}$.

For $0 \leq i \leq n$, consider the family of endomorphisms $f_{i}: \mathbb{P}_{k}^{n} \times \mathbb{A}_{k}^{n} \rightarrow \mathbb{P}_{k}^{n} \times \mathbb{A}_{k}^{n}$ given by

$$
\begin{aligned}
& f_{i}\left(\left(\left[x_{0}, \ldots, x_{i}, \ldots, x_{n}\right],\left(c_{1}, c_{2}, \ldots, c_{n}\right)\right)\right) \\
& =\left(\left[x_{0}^{d}+c_{1} x_{i}^{d}, \ldots, x_{i-1}^{d}+c_{i} x_{i}^{d}, x_{i}^{d}, x_{i+1}^{d}+c_{i+1} x_{i}^{d}, \ldots, x_{n}^{d}+c_{n} x_{i}^{d}\right],\left(c_{1}, c_{2}, \ldots, c_{n}\right)\right) .
\end{aligned}
$$

On the open affine $U_{i}$ given by $x_{i} \neq 0, f_{i}$ is the product of the $n$ polynomials $\phi_{c_{j}}$. On the complement of this affine, i.e., on the subvariety given by $x_{i}=0$ (which is also preserved by $f_{i}$ ), the maps do not depend on $c_{j}$, so the monodromy action of this family on the periodic points in this locus is trivial. Let $G_{i}$ be the subgroup of the monodromy group acting on $\operatorname{Per}_{g_{n, d}}$ corresponding to this family; by applying Theorem 3.2, one gets a complete description of this group. We let $G$ be the subgroup of the monodromy group generated by all the $G_{i}$.

Let $P=[\zeta, 1, \ldots, 1,1]$ where $\zeta$ is in $\operatorname{Per}_{\phi_{0}}(b)$, and let $Q=\left[\xi_{0}, \xi_{1}, \ldots, \xi_{n}\right]$ be any other element of $\operatorname{Per}_{g_{n, d}}(b)$. We may assume that some $\xi_{i}=1$, so each $\xi_{j} \in \operatorname{Per}_{\phi_{0}}\left(b^{\prime}\right)$ for some $b^{\prime} \mid b$, and also the lcm of the periods of all the $\xi_{j}$ is $b$. We prove the transitivity of the monodromy action by showing that there exists an element in the monodromy that sends $P$ to $Q$.

From the transitivity of the Galois action in Theorem 3.2, it follows that for $\xi_{j} \in \operatorname{Per}_{\phi_{0}}\left(b^{\prime}\right)$, as long as some $\xi_{i}=1$ with $i \neq j$, we can find an element of $G$ that fixes all coordinates of $Q$ except that it replaces $\xi_{j}$ with any other $\xi_{j}^{\prime} \in \operatorname{Per}_{\phi_{0}}\left(b^{\prime}\right)$. Since $0,1 \in \operatorname{Per}_{\phi_{0}}(0)$, we may use this to assume that all $\xi_{i} \neq 0$ and then also, by dividing through by $\xi_{n}$, that $\xi_{n}=1$.

We now show that we may also assume that $\xi_{0} \in \operatorname{Per}_{\phi_{0}}(b)$. Suppose $\xi_{0}$ is a primitive $m$-th root of unity, $\xi_{1}$ is a primitive $m^{\prime}$-th root of unity and $\xi_{0} \in \operatorname{Per}_{\phi_{0}}(a)$ and $\xi_{1} \in \operatorname{Per}_{\phi_{0}}\left(a^{\prime}\right)$. By using the action of $G$, we may change $\xi_{0}$ and $\xi_{1}$ so that $m=d^{a}-1$ and $m^{\prime}=d^{a^{\prime}}-1$. If the highest powers of 2 dividing $m$ and $m^{\prime}$ are equal and greater than 1 , we may change $\xi_{0}$ to a primitive $\left(d^{a}-1\right) / 2$-th root of unity; the period $a$ remains unchanged. By Lemma 3.4, we may then assume that $\xi_{0} \xi_{1}{ }^{-1}$ is a primitive $\operatorname{lcm}\left(m, m^{\prime}\right)$-th root of unity. We multiply all coordinates of $Q$ by $\xi_{1}^{-1}$, so the zeroth coordinate becomes $\xi_{0} \xi_{1}^{-1}$, and the second becomes 1 . Using the action of $G$, we then replace the zeroth coordinate by $\eta$ as in Lemma 3.4 while keeping all other coordinates fixed. We then multiply all coordinates by $\xi_{1}$. The resulting point 
has all coordinates except for the zeroth equal to the corresponding coordinates of $Q$ while the zeroth coordinate is now in $\operatorname{Per}_{\phi_{0}}\left(\operatorname{lcm}\left(a, a^{\prime}\right)\right)$. Repeating this procedure with $\xi_{1}$ replaced by $\xi_{2}$, then $\xi_{3}$ and so on, since the lcm of the periods of all the $\xi_{i}$ is $b$, it follows that eventually we have that $\xi_{0} \in \operatorname{Per}_{\phi_{0}}(b)$.

We now inductively transform $P$ into $Q$ using the action of $G$. If $\xi_{i}$ has period $a_{i}$ as an element of $\operatorname{Per}_{\phi_{0}}$, we may use the action of $G$ to replace it by a primitive $\left(d^{a_{i}}-1\right)$-th root of unity. If the highest power of 2 dividing $d^{a_{i}}-1$ is equal to the highest power of 2 dividing $d^{b}-1$ and $d$ is odd, then we use a primitive $\left(d^{a_{i}}-1 / 2\right)$-th root of unity instead if $i>0$.

Let $P_{0}=P$, and suppose we have constructed $P_{i}=\left[\xi_{0, i}, \xi_{1, i}, \ldots, \xi_{n-1, i}, 1\right]$ in $\operatorname{Per}_{\phi_{0}}(b)$ by induction, with the following properties:

(1) $\xi_{0, i} \in \operatorname{Per}_{\phi_{0}}(b)$.

(2) $\xi_{j, i}=\xi_{j}$ for $0<j \leq i$.

(3) $\xi_{j, 1}=1$ for $j>i$.

Clearly $P_{0}$ satisfies these properties; we will show that given $P_{i}$ with $i<n$ we can find an element of $G$ that transforms it into a point $P_{i+1}$ with the required properties.

So suppose $P_{i}$ has been constructed. By Lemma 3.4, there exists $\eta \in \operatorname{Per}_{\phi_{0}}(b)$ so that $\eta^{-1} \xi_{i+1} \in \operatorname{Per}_{\phi_{0}}(b)$. Since $\xi_{0, i} \in \operatorname{Per}_{\phi_{0}}(b)$, we may use the action of $G$ to replace $\xi_{0, i}$ with $\eta$ while keeping the other coordinates fixed. We then multiply all coordinates by $\eta^{-1}$, so the zeroth coordinate becomes 1 , and the $(i+1)$-th coordinate becomes $\eta^{-1}$. Since $\eta^{-1} \xi_{i+1} \in \operatorname{Per}_{\phi_{0}}(b)$, we may use the action of $G$ to replace the $(i+1)$-th coordinate by $\eta^{-1} \xi_{i+1}$ while keeping all the other coordinates fixed. If we now multiply all coordinates by $\eta$, we obtain a point $P_{i+1}$ with the property that all coordinates except the zeroth and $(i+1)$-th of $P_{i}$ and $P_{i+1}$ are equal, the zeroth coordinate is $\eta \in \operatorname{Per}_{\phi_{0}}(b)$ and the $(i+1)$-th is $\xi_{i+1}$, so fulfilling the requirements.

We thus obtain a point $P_{n}$ with the property that all coordinates of $P_{n}$ and $Q$ are equal except possibly for the first. Since the zeroth coordinate of both is in $\operatorname{Per}_{\phi_{0}}(b)$ and the $n$-th is 1 , we may use an element of $G$ to transform $P_{n}$ into $Q$. It follows that the action of $G$, hence of the full monodromy group, is transitive on $\operatorname{Per}_{\phi_{0}}(b)$.

\section{Corollary 3.5. No preperiodic point of $f_{n, d}$ lies in the ramification locus.}

Proof. The ramification locus of $f_{n, d}$ is defined over $k_{n, d}$; thus, if one preperiodic point lies in the ramification locus, so must its entire Galois orbit.

The Galois orbit must map onto the Galois orbit of the corresponding periodic point, i.e., the periodic point $y$ such that $f_{n, d}^{r}(x)=y$ and $f_{n, d}^{s}(x)$ is not periodic for any $s<r$. But this orbit consists of all periodic points of a fixed period $b$ by Proposition 3.3. By specialisation to the $d$-power map $g_{n, d}$, we see that this is not possible: 
the ramification locus of this map is actually preserved by the map and for no period $b$ are all the periodic points of period $b$ contained in the ramification locus.

Theorem 3.6. Let $f: \mathbb{P}_{K}^{n} \rightarrow \mathbb{P}_{K}^{n}$ be a generic endomorphism of degree $d>1$ over an algebraically closed field $K$ of characteristic zero. Then:

(1) If $X \subset \mathbb{P}_{K}^{n}$ is an irreducible subvariety such that $f^{r}(X)=X$ for some $r>0$, then $X$ is a point or $X=\mathbb{P}_{K}^{n}$.

(2) If $O_{f}(x)$ is infinite for $x \in \mathbb{P}^{n}(K)$, then $O_{f}(x)$ is Zariski-dense in $\mathbb{P}_{K}^{n}$.

Proof. Since $f$ is generic, we may identify $f$ with $f_{n, d}$ defined above. Any specialisation of $X$ to a subvariety of $\mathbb{P}^{n}$ defined over a finite extension of $k_{n, d}$ satisfies the same property as $X$, so we may assume that $X$ is defined over a finite extension of degree $m$ of $k_{n, d}$. Replacing $X$ by the union of its Galois conjugates, we may assume that $X$ is defined over $k_{n, d}$. Since $\operatorname{dim}(X)>0$, it follows by [Fakhruddin 2003, Theorem 5.1] applied to $f^{r}$ that $X$ contains infinitely many periodic points of $f$. Since $X$ is defined over $k_{n, d}$, it then follows from Proposition 3.3 that there is an infinite sequence of distinct integers $b_{i}, i=1,2, \ldots$, such that $X$ contains all the periodic points of $f_{n, d}$ of period $b_{i}$ for all $i$. Let $g_{n, d}: \mathbb{P}_{k}^{n} \rightarrow \mathbb{P}_{k}^{n}$ be, as before, the map that raises each coordinate to its $d$-th power. By specialisation and Lemma 2.2(2), we obtain a subvariety $X^{\prime}$ of $\mathbb{P}_{k}^{n}$ defined over $k$ that contains all the periodic points of $g_{n, d}$ of period $b_{i}$ for all $i$.

For $Z \subset \mathbb{A}_{k}^{n}$ any subvariety (with $k$ any field), if there is a sequence of finite subsets $S_{i} \subset \mathbb{A}^{n}(\bar{k})$ such that $\left(S_{i}\right)^{n} \subset Z(\bar{k})$ for all $i$ and $\left|S_{i}\right| \rightarrow \infty$, then $Z=\mathbb{A}_{k}^{n}$; this is well-known and follows, for example, from [Tao and $\mathrm{Vu} 2006$, Theorem 9.2]. By the previous paragraph, for all $i, X^{\prime}$ contains all elements of $\mathbb{P}^{n}(\bar{k})$ of the form $\left[\xi_{0}, \ldots, \xi_{n-1}, 1\right]$ with $\xi_{j} \in \operatorname{Per}_{\phi_{0}}\left(b_{i}\right)$. Since $\left|\operatorname{Per}_{\phi_{0}}\left(b_{i}\right)\right| \rightarrow \infty$ with $i$, we must have $X^{\prime}=\mathbb{P}_{k}^{n}$ and hence $X=\mathbb{P}_{k_{n, d}}^{n}$, proving (1).

To prove (2), we observe that the Zariski closure of $O_{f}(x)$ is mapped into itself by $f$. If it is infinite, it must contain a positive-dimensional subvariety $X$ such that $f^{r}(X)=X$ for some $r>0$. By (1), we must have $X=\mathbb{P}_{K}^{n}$.

Remark 3.7. One can give a simpler proof of (1) using the Lefschetz trace formula rather than [Fakhruddin 2003, Theorem 5.1] if one only considers smooth $X$. Also, from Theorem 4.1, it follows that the elementary version of [Fakhruddin 2003, Theorem 5.1] asserting the density of preperiodic points suffices, but this introduces other dependencies.

\section{The dynamical "Manin-Mumford" conjecture for generic endomorphisms}

The original "Manin-Mumford" conjecture of Zhang asserted that, for any polarised endomorphism $f$ of a projective variety $X$ over a field $K$ of characteristic 0 , any subvariety $Y$ of $X$ containing a Zariski-dense set of preperiodic points is preperiodic. 
This was known for abelian varieties and the multiplication-by- $m$ maps but was later shown to be false in general, even if $X=\mathbb{P}_{K}^{n}$, by Ghioca and Tucker. Ghioca, Tucker and Zhang then proposed a modified conjecture that takes into account the action of $f$ on the tangent space; see the article [Ghioca et al. 2011] for a discussion of the history, the statement of the modified version and some positive results. ${ }^{2}$

The following theorem implies that Zhang's conjecture, in its original form, holds for generic endomorphisms of $\mathbb{P}_{K}^{n}$ :

Theorem 4.1. For $f: \mathbb{P}_{K}^{n} \rightarrow \mathbb{P}_{K}^{n}$ a generic endomorphism of degree $d>1$ over an algebraically closed field $K$ of characteristic zero, any infinite subset of $\mathbb{P}^{n}(K)$ consisting of $f$-preperiodic points is Zariski-dense in $\mathbb{P}_{K}^{n}$.

As in the other results of this paper, one of the key ingredients of the proof is the Galois action on the set of periodic points. However, Proposition 3.3 alone does not suffice since we also need the transitivity of the monodromy action on the set of preperiodic points. It turns out that transitivity does not hold for the monodromy action on $\operatorname{Prep}_{f}(a, b)$ for $f$ as in Theorem 3.2 and $d>2$. Nevertheless, by considering a larger family of polynomials, we prove in Proposition 4.6 that the monodromy action on $\operatorname{Prep}_{f_{n, d}}(a, b)$ is indeed transitive for all $a$ and $b$. This then allows us to use a specialisation argument to prove Theorem 4.1.

Fix $d>1$. For $b>0$, consider the polynomial $P_{b}(c)=\phi_{c}^{b}(0)$. The roots of $P_{b}(c)$ are exactly the parameters $c$ so that 0 is a periodic point of period dividing $b$ for the polynomial $\phi_{c}(z)$.

Lemma 4.2 (Gleason). All roots of $P_{b}$ are multiplicity-free.

Proof. The proof given in [Douady and Hubbard 1985, Lemma 19.1] for $d=2$ goes through for general $d$ simply by replacing 2 by $d$.

Lemma 4.3. Fix $d>1$. For $(c, \epsilon) \in \mathbb{A}^{2}$, let $\phi_{c, \epsilon}: \mathbb{A}^{1} \rightarrow \mathbb{A}^{1}$ be given by $\phi_{c, \epsilon}(z)=$ $z(z-\epsilon)^{d-1}+c$. Then the monodromy action on $\operatorname{Prep}_{\phi_{c, \epsilon}}(1, b)$ is transitive for all $b>0$.

Proof. It follows from Theorem 3.2 that the monodromy action on $\operatorname{Per}_{\phi_{c, \epsilon}}(b)$ is transitive for all $b>0$, so it suffices to prove that for any $b>0$ there exists $x \in \operatorname{Per}_{\phi_{c, \epsilon}}(b)$ and an element $\gamma$ of the monodromy so that $\gamma(x)=x$ and $\gamma$ cyclically permutes the $d-1$ elements of $\phi_{c, \epsilon}^{-1}(x)$ (so if $d=2$, there is nothing to prove).

By Lemma 4.2 and a simple counting argument, it follows that, for any $b>0$, there exists $c_{b} \in \mathbb{C}$ so that $0 \in \operatorname{Per}_{\phi_{c_{b}}}(b)$. Since 0 is a critical point of $\phi_{c_{b}}$, it is of multiplicity 1 as an element of $\operatorname{Per}_{\phi_{c_{b}}}(b)$. It follows that, for $|\epsilon| \ll 0$, there exists $c_{b, \epsilon}$ close to $c_{b}$ so that 0 is a periodic point of $\operatorname{Per}_{\phi_{c_{b, \epsilon}, \epsilon}}(b)$ of multiplicity 1 . By the

${ }^{2}$ It seems reasonable to expect the modified conjecture to hold even for quasipolarised endomorphisms. 
definition of $\phi_{c, \epsilon}$, it then follows that $\epsilon$ is the unique element of $\operatorname{Prep}_{\phi_{c_{b, \epsilon}, \epsilon}}(1, b)$ that is mapped to $\phi_{c_{b, \epsilon}, \epsilon}(0)=c_{b, \epsilon}$ by $\phi_{c_{b, \epsilon}, \epsilon}$.

We now consider the 1-parameter family of maps $\phi_{c, \epsilon}$ with $\epsilon$ such that $|\epsilon| \ll 0$ fixed. In a neighbourhood of $c_{b, \epsilon}$, the element $c_{b, \epsilon} \in \operatorname{Per}_{\phi_{c_{b, \epsilon}, \epsilon}}(b)$ deforms uniquely as an element of $\operatorname{Per}_{\phi_{c, \epsilon}}(b)$. However, since the critical points of $\phi_{c, \epsilon}$ are independent of $c$, it follows that the element $\epsilon \in \operatorname{Prep}_{\phi_{c_{b, \epsilon}, \epsilon}}(1, b)$ deforms to $d-1$ distinct elements of $\operatorname{Prep}_{\phi_{c, \epsilon}}(1, b)$ that are all mapped to the deformed periodic point above by $\phi_{c, \epsilon}$.

We claim that the monodromy action of a small loop around $c_{b, \epsilon}$ gives us the required element $\gamma$. Since $c_{b, \epsilon}$ deforms uniquely as a periodic point, the monodromy action of $\gamma$ on this point is trivial as required. To prove that the second condition is satisfied, let $C \subset \mathbb{A}^{2}$ be the curve consisting of all points $(z, c)$ so that $z$ is a preperiodic point of preperiod 1 and period $b$ of $\phi_{c, \epsilon}$ (with $\epsilon$ fixed as above). It suffices to prove that $C$ is smooth at the point $\left(\epsilon, c_{b, \epsilon}\right)$.

To see this, we consider the explicit equation for $C$. It is given in an neighbourhood of $\left(\epsilon, c_{b, \epsilon}\right)$ by

$$
\phi_{c, \epsilon}^{b+1}(z)-\phi_{c, \epsilon}(z)=0 .
$$

To see that it is smooth at $\left(c_{b, \epsilon}, \epsilon\right)$, it suffices to substitute $\epsilon$ for $z$ and check that the resulting polynomial in $c, \phi_{c, \epsilon}^{b+1}(\epsilon)-\phi_{c, \epsilon}(\epsilon)$, has $c_{b, \epsilon}$ as a root of multiplicity 1 . However, from the definition of $\phi_{c, \epsilon}$ it follows that

$$
\phi_{c, \epsilon}^{b+1}(\epsilon)-\phi_{c, \epsilon}(\epsilon)=\phi_{c, \epsilon}^{b+1}(0)-\phi_{c, \epsilon}(0),
$$

so we may replace $\epsilon$ by 0 . In a neighbourhood of the point $\left(0, c_{b, \epsilon}\right)$, the curve given by (4-1) is smooth since it parametrises periodic points of period $b$ and the periodic point 0 of $\phi_{c_{b, e}, \epsilon}$ is of multiplicity 1 . To show that the multiplicity of $c_{b, \epsilon}$ as a root of $\phi_{c, \epsilon}^{b+1}(0)-\phi_{c, \epsilon}(0)$ is 1 , we may then specialise $\epsilon$ to 0 , so it suffices to consider the multiplicity of $c_{b}$ as a root of the polynomial $P_{b}(c)=\phi_{c}^{b}$. By Lemma 4.2, this multiplicity is indeed 1 as required.

To prove the transitivity of the monodromy action on $\operatorname{Prep}_{\phi_{c, \epsilon}}(a, b)$ for $a>1$, we shall need some results about Misiurewicz points. We refer the reader to [Lau and Schleicher 1994; Eberlein 1999] for the basic facts that we use below, which generalise results proved in [Douady and Hubbard 1984] in the case $d=2$. Recall that $c_{0} \in \mathbb{C}$ is called a Misiurewicz point if $c_{0}$ is a strictly preperiodic point of the map $\phi_{c_{0}}$. By the results of [op. cit.], for any strictly preperiodic angle $\theta \in \mathbb{Q} / \mathbb{Z}$, there is a Misiurewicz point $c_{0}$ such that the parameter ray with angle $\theta$ lands at $c_{0}$. By [Eberlein 1999, Lemma 8.3], the preperiod of $\theta$ (with respect to multiplication by $d$ ) is equal to the preperiod of $c_{0}$ (with respect to $\phi_{c_{0}}$ ) and the period of the kneading sequence of $\theta, K(\theta)$, is equal to the period of $c_{0}$ (with respect to $\phi_{c_{0}}$ ). For $\theta=1 /\left(d^{a} \cdot\left(d^{b}-1\right)\right), a, b>0$, the preperiod of $\theta$ is $a$ and the period of $K(\theta)$ is $b$, so there exists a Misiurewicz point with any preperiod $a>0$ and period $b$. 
A point $\lambda \in \mathbb{C}$ is called parabolic of period $b$ if it is the landing point of a parameter ray with angle $\theta$ that is periodic of period $b$. By results from [Douady and Hubbard 1984; Eberlein 1999], a parabolic point is never a Misiurewicz point.

To prove the transitivity of the monodromy action for $a>1$, we shall need the following analogue of Lemma 4.2, due to Douady and Hubbard:

Lemma 4.4. For a Misiurewicz point $c_{0}$ as above, the equation $\phi_{c}^{a+1+b}(0)-$ $\phi_{c}^{a+1}(0)=0$ has a simple root at $c=c_{0}$.

Proof. The lemma is formulated and proved for $d=2$ as Corollary 8.5 of [Douady and Hubbard 1984]; however, Proposition 8.5 of the same work holds for general $d$, and so the proof goes through if we substitute Theorems 8.1 and 8.2 of [Eberlein 1999] for Douady and Hubbard's Theorem 8.2.

Lemma 4.5. The monodromy action of the 2-dimensional family of polynomials $\phi_{c, \epsilon}$ on $\operatorname{Prep}_{\phi_{c, \epsilon}}(a, b)$ is transitive for all $a, b>0$.

Proof. We already know transitivity if $a=1$. Thus, by induction, we may assume $a>1$, and then it suffices to prove that for the 1-dimensional family of polynomials $\phi_{c}$, there exists $x \in \operatorname{Per}_{\phi_{c}}(a-1, b)$ and an element $\gamma$ of the monodromy such that $\gamma(x)=x$ and $\gamma$ induces a cyclic permutation on the $d$ elements of $\operatorname{Per}_{\phi_{c}}(a-1, b)$ comprising $\phi_{c}^{-1}(x)$.

Since 0 and $\infty$ are the only critical points of $\phi_{\lambda}$, the preperiodic points for general $\lambda$ are multiplicity-free. Let $c$ be a Misiurewicz point of preperiod $a-1$ and period $b$, and consider a small loop $\gamma$ in the parameter plane around $c$ such that all parabolic points of period $b$ and all Misiurewicz points of preperiod $a$ and period $b$ are outside this loop. We note that the preperiodic points of $\phi_{c}$ of preperiod $\leq a$ and period $b$ are multiplicity-free since none of them are critical values and a Misiurewicz point is never a parabolic point. This remains true in a neighbourhood of $c$, so we may assume that this holds in a neighbourhood $U$ of $\gamma$ containing its interior; in particular, $c$ deforms uniquely as a preperiodic point $c_{\lambda} \in \operatorname{Prep}_{\phi_{\lambda}}(a, b)$ as $\lambda$ varies in this neighbourhood.

Since $\phi_{c}$ is totally ramified at 0,0 is the unique element of $\operatorname{Prep}_{\phi_{c}}(a, b)$ mapping to $c$ by $\phi_{c}$. By construction, for any other $\lambda \in U$, there are $d$ points of $\operatorname{Prep}_{\phi_{\lambda}}(a, b)$ mapping to $c_{\lambda}$ and these points all come together at 0 as $\lambda \rightarrow c$. The set of these points in a neighbourhood of $(c, 0)$ is exactly the zero locus $D$ of the polynomial $\phi_{\lambda}^{a+1+b}(z)-\phi_{\lambda}^{a+1}(z)$. By Lemma 4.4, the multiplicity of this after setting $z=0$ is 1 at $(c, 0)$, so it follows that $D$ must be smooth at this point. Since the inverse image of $c$ in $D$ is a single point, it follows that the map induced by the projection to the first factor is totally ramified of degree $d$ at $(c, 0)$. Consequently, the monodromy around $\gamma$ induces a cyclic permutation of order $d$ on $\phi_{\lambda}^{-1}\left(c_{\lambda}\right)$. We may thus take $x=c_{\lambda}$ for any $\lambda$ with $|\lambda| \ll 0$ to complete the proof. 
Proposition 4.6. The Galois action on $\operatorname{Prep}_{f_{n, d}}(a, b)$ is transitive for all $a, b>0$.

Proof. Since $f_{n, d}$ is defined over $k_{n, d}$, the Galois action on $\operatorname{Prep}_{f_{n, d}}(a, b), a, b \geq 1$, is compatible with the natural surjections

$$
\operatorname{Prep}_{f_{n, d}}(a+1, b) \stackrel{f_{n, d}}{\longrightarrow} \operatorname{Prep}_{f_{n, d}}(a, b) \text {. }
$$

Thus, by induction on $a$, it suffices to show that for any $a>0$, there exists an element $x \in \operatorname{Prep}_{f_{n, d}}(a-1, b)$ such that, for any $y, y^{\prime} \in \operatorname{Prep}_{f_{n, d}}(a, b)$ with $f_{n, d}(y)=f_{n, d}\left(y^{\prime}\right)$, there exists an element $\gamma$ of the monodromy such that $\gamma(y)=y^{\prime}$.

If $n=1$, the claim follows immediately from Lemma 4.5, so in the following, we shall assume $n>1$.

As before, we may assume that $k=\mathbb{C}$. The proof of transitivity is similar to that for the case of periodic points except that we replace the use of the maps $\phi_{c}$ by $\phi_{c, \epsilon}$. So for $0 \leq i \leq n$, consider the family of endomorphisms $f_{i}: \mathbb{P}^{n} \times \mathbb{A}^{2 n} \rightarrow \mathbb{P}^{n} \times \mathbb{A}^{2 n}$ defined by

$$
\begin{aligned}
f_{i}\left(\left(\left[x_{0}, \ldots, x_{n}\right],\left(c_{1}, \ldots, c_{n}, \epsilon_{1}, \ldots, \epsilon_{n}\right)\right)\right) & \\
= & \left(\left[x_{0}\left(x_{0}-\epsilon_{1} x_{i}\right)^{d-1}+c_{1} x_{i}^{d}, \ldots, x_{i-1}\left(x_{i-1}-\epsilon_{i} x_{i}\right)^{d-1}+c_{i} x_{i}^{d}, x_{i}^{d},\right.\right. \\
& \left.x_{i+1}\left(x_{i+1}-\epsilon_{i+1} x_{i}\right)^{d-1}+x_{i}^{d}, \ldots, x_{n}\left(x_{n}-\epsilon_{n} x_{i}\right)^{d-1}+c_{n} x_{i}^{d}\right], \\
& \left.\left(c_{1}, \ldots, c_{n}, \epsilon_{1}, \ldots, \epsilon_{n}\right)\right) .
\end{aligned}
$$

On the open affine $U_{i}$ given by $x_{i} \neq 0, f_{i}$ is the product of the $n$ polynomials $\phi_{c_{j}, \epsilon_{j}}$.

Let $g_{n, d}$ be the $d$-power map as before. Contrary to the case of periodic points, the map from the locus of preperiodic points to the base is not étale at preperiodic points of $g_{n, d}$ contained in its ramification locus. However, the map is étale at preperiodic points all of whose coordinates are nonzero, and this will suffice (except when $d=2$ ) for our needs (compare the discussion of monodromy on page 589).

Suppose $a=1$. Let $\zeta$ be a primitive $\left(d^{b}-1\right)$-th root of unity, and let $x^{\prime} \in \operatorname{Per}_{g_{n, d}}(b)$ be the point $[\zeta, 1, \ldots, 1,1]$, and let $x=g_{n, d}\left(x^{\prime}\right)$. The preperiodic points $y$ such that $g_{n, d}(y)=x$ are of the form $\left[\zeta \xi_{1}, \xi_{2}, \ldots, \xi_{n}, 1\right]$, where all the $\xi_{i}$ are $d$-th roots of unity and at least one of them is not equal to 1 .

We now also assume that $d>2$. Using the monodromy action of the family $f_{n}$ and Lemma 4.3, it follows that we may assume that $\xi_{i}=\xi$, where $\xi$ is a fixed $d$-th root of unity or $\xi_{i}=1$.

Since $d>1$, there exists a $d$-th root of unity $\xi^{\prime}$ such that $\xi^{\prime} \neq 1, \xi$. Let $y=$ $[\zeta \xi, 1, \ldots, 1]$ and $y^{\prime}=[\zeta \xi, \xi, \ldots, \xi, 1, \ldots, 1]$, where there are $n_{1} \xi$ 's and $n_{2} 1$ 's with $n_{1}, n_{2}>0$. Each step of the following sequence of transformations is given either by multiplying through by a constant or by applying the monodromy of $f_{i}$ for some $i$ such that the $i$-th coordinate is equal to 1 : 


$$
\begin{aligned}
y=[\zeta \xi, 1, \ldots, 1] & \rightarrow\left[1, \zeta^{-1} \xi^{-1}, \ldots, \zeta^{-1} \xi^{-1}\right] \\
& \rightarrow\left[1, \zeta^{-1} \xi^{\prime-1}, \ldots, \zeta^{-1} \xi^{\prime-1}, \zeta^{-1} \xi^{-1}, \ldots, \zeta^{-1} \xi^{-1}\right] \\
& \rightarrow\left[\zeta \xi, \xi \xi^{\prime-1}, \ldots, \xi \xi^{\prime-1}, 1 \ldots, 1\right] \\
& \rightarrow[\zeta \xi, \xi, \ldots, \xi, 1, \ldots, 1]=y^{\prime} .
\end{aligned}
$$

In the last transformation, we also use the fact that $\xi \xi^{\prime-1}$ is a $d$-th root of unity not equal to 1 so, like $\xi$ and $\xi^{\prime}$, an element of $\operatorname{Prep}_{\phi_{0}}(1,1)$.

Let $y^{\prime \prime}=[\zeta, \xi, \ldots, \xi, 1 \ldots, 1]$, where there are $n_{1} \xi$ 's and $n_{2}$ 1's with $n_{1}, n_{2}>0$ as before. Since $d>2$, there exists $\zeta^{\prime} \in \operatorname{Per}_{\phi_{0}}(b)$ such that $\zeta \zeta^{\prime-1}$ also has period $b$. We then have a similar sequence of transformations:

$$
\begin{aligned}
y^{\prime}=[\zeta \xi, \xi, \ldots, \xi, 1, \ldots, 1] & \rightarrow\left[1, \zeta^{-1}, \ldots, \zeta^{-1}, \zeta^{-1} \xi^{-1}, \ldots, \zeta^{-1} \xi^{-1}\right] \\
& \rightarrow\left[1, \zeta^{\prime} \zeta^{-1}, \ldots, \zeta \zeta^{-1}, \zeta^{-1} \xi^{-1}, \ldots, \zeta^{-1} \xi^{-1}\right] \\
& \rightarrow\left[\zeta \xi, \zeta^{\prime} \xi, \ldots, \zeta^{\prime} \xi, 1, \ldots, 1\right] \\
& \rightarrow\left[\zeta \xi, \zeta^{\prime} \xi^{\prime}, \ldots, \zeta^{\prime} \xi^{\prime}, 1, \ldots, 1\right] \\
& \rightarrow\left[\zeta \xi\left(\zeta^{\prime} \xi^{\prime}\right)^{-1}, 1, \ldots, 1,\left(\zeta^{\prime} \xi^{\prime}\right)^{-1}, \ldots,\left(\zeta^{\prime} \xi^{\prime}\right)^{-1}\right] \\
& \rightarrow\left[\zeta\left(\zeta^{\prime} \xi\right)^{-1}, 1, \ldots, 1,\left(\zeta^{\prime} \xi\right)^{-1}, \ldots,\left(\zeta^{\prime} \xi\right)^{-1}\right] \\
& \rightarrow\left[\zeta, \zeta^{\prime} \xi, \ldots, \zeta^{\prime} \xi, 1, \ldots, 1\right] \\
& \rightarrow\left[1, \zeta^{-1} \zeta^{\prime} \xi, \ldots, \zeta^{-1} \zeta^{\prime} \xi, \zeta^{-1}, \ldots, \zeta^{-1}\right] \\
& \rightarrow\left[1, \zeta^{-1} \xi, \ldots, \zeta^{-1} \xi, \zeta^{-1}, \ldots, \zeta^{-1}\right] \\
& \rightarrow[\zeta, \xi, \ldots, \xi, 1, \ldots, 1]=y^{\prime \prime} .
\end{aligned}
$$

Here we have used that $\zeta, \zeta^{\prime}, \zeta \zeta^{\prime-1}$ and their inverses are in $\operatorname{Per}_{\phi_{0}}(b)$ and each one of these multiplied by $\xi, \xi^{\prime}, \xi \xi^{-1}$ or any of their inverses is an element of $\operatorname{Prep}_{\phi_{0}}(1, b)$. By symmetry, we then conclude that the desired transitivity holds in this case.

Now suppose $d=2$. In this case, $\operatorname{Prep}_{\phi_{0}}(1,1)=\{-1\}$, a singleton, so the above argument breaks down, and we will need to consider paths passing through elements of $\operatorname{Prep}_{g_{n, d}}(1, b)$, one of whose coordinates is 0 . This is justified by Lemma 4.7 below.

As before, let $y=[-\zeta, \ldots,-\zeta, 1]$ with $\zeta$ a primitive $\left(2^{b}-1\right)$-th root of unity and $y^{\prime}=[-\zeta, \ldots,-\zeta, \zeta, \ldots, \zeta, 1]$, where there is at least one $-\zeta$ and one $\zeta$. We need to consider the cases $b=1$ and $b>1$ separately.

First suppose $b=1$, so $\zeta=1$. We then have the sequence of transformations

$$
\begin{aligned}
y=[-1, \ldots,-1,1] & \rightarrow[1, \ldots, 1,-1] \rightarrow[1, \ldots, 1,0,-1] \\
& \rightarrow[-1, \ldots,-1,0,1] \rightarrow[-1, \ldots,-1,1,1] .
\end{aligned}
$$


Repeating this procedure, we see that we can connect $y$ to $y^{\prime}$, and by symmetry, the transitivity follows in this case.

Now suppose $b>1$, so there exists $\zeta^{\prime} \in \operatorname{Per}_{\phi_{0}}(b)$ so that, as before, $\zeta \zeta^{\prime-1} \in$ $\operatorname{Per}_{\phi_{0}}(b)$. We then have a sequence of transformations

$$
\begin{aligned}
y=[-\zeta, \ldots,-\zeta, 1] & \rightarrow\left[1, \ldots, 1,-\zeta^{-1}\right] \rightarrow\left[1, \ldots, 1,0,-\zeta^{-1}\right] \\
& \rightarrow[-\zeta, \ldots,-\zeta, 0,1] \rightarrow[-\zeta, \ldots,-\zeta, 1,1] \\
& \rightarrow\left[1, \ldots, 1,-\zeta^{-1},-\zeta^{-1}\right] \rightarrow\left[1, \ldots, 1,-\zeta^{\prime-1},-\zeta^{-1}\right] \\
& \rightarrow\left[-\zeta^{-1}, \ldots,-\zeta^{-1}, \zeta \zeta^{\prime-1}, 1\right] \rightarrow\left[-\zeta^{-1}, \ldots,-\zeta^{-1}, \zeta, 1\right] .
\end{aligned}
$$

Repeating this procedure, we see that we can connect $y$ to $y^{\prime}$, and then by symmetry, transitivity follows.

Finally, suppose $a>1$. By induction, we can choose $x$ to be an arbitrary point of preperiod $a-1$ and period $b$, so we let $x^{\prime}=[\zeta, \zeta, \ldots, \zeta, 1]$ where $\zeta$ is an element of $\operatorname{Prep}_{\phi_{0}}(a, 1)$ and $x=g_{n, d}(x)$. The points in $g_{n, d}^{-1}(x)$ are of the form $\left[\zeta \xi_{1}, \ldots, \zeta \xi_{n}, 1\right]$ where $\xi_{1}$ is a $d$-th root of 1 , so $\zeta \xi_{i} \in \operatorname{Prep}_{\phi_{0}}(a, 1)$. One sees that the monodromy acts transitively on $g_{n, d}^{-1}(x)$ simply by considering the monodromy of the family of maps $f_{n}$ and applying Lemma 4.5.

Let $F: \mathbb{P}_{k}^{n} \times \operatorname{Mor}_{n, d / k} \rightarrow \mathbb{P}_{k}^{n} \rightarrow \operatorname{Mor}_{n, d / k}$ be the universal morphism of degree $d$, and consider $P_{F}(b+1, b) \subset \operatorname{Mor}_{n, d / k}$ (the notation is defined just before Lemma 2.2). The fibre of the projection map from $P_{F}(b+1, b)$ to Mor $_{n, d / k}$ over any point $f \in \operatorname{Mor}_{n, d / k}$ consists of $f$-preperiodic points of preperiod at most 1 and period dividing $b$.

Lemma 4.7. If $d=2$ and $\operatorname{char}(k) \neq 2$, then $P_{F}(b+1, b)$ is smooth at any preperiodic point of $g_{n, 2}$ of preperiod 1 and period $b$.

Proof. We have $F\left(P_{F}(b+1, b)\right)=P_{F}(b)$. As we have already seen, $P_{F}(b)$ is smooth at all periodic points of $g_{n, d}$. Moreover, $F$ is analytically locally at any point of $\operatorname{Prep}_{g_{n, d}}(1, b)$ with exactly one coordinate equal to 0 , a cyclic cover of degree 2. Thus, to prove smoothness, it suffices to show that the discriminant of $F$ intersects $P_{F}(b)$ transversely at any point of $\operatorname{Per}_{g_{n, d}}(b)$ with exactly one coordinate equal to 0 .

To prove transversality, it then suffices to restrict to any subvariety of $\operatorname{Mor}_{n, d}$ and prove transversality for the induced subvarieties. By considering, say, the family $f_{i}$ as in the proof of Proposition 3.3, where $i$ is such that the $i$-th coordinate of the point under consideration is nonzero, we reduce to the case of the one parameter $\phi_{c}, c \in \mathbb{A}_{c}^{1}$, and we need to prove transversality at the point $(z, c)=(0,0)$. The discriminant locus is given by $z=0$ (since $d=2$ ) and the locus of fixed points by $z^{2}+c-z=0$, so the lemma follows. 
Proof of Theorem 4.1. Let $X$ be the Zariski closure of an infinite subset of preperiodic points. By Lemma 2.2(1), all preperiodic points of a quasipolarised map are defined over the algebraic closure of the base field, so we may assume without loss of generality that $K$ is the algebraic closure of $k_{n, d}$ and $X$ is defined over a finite extension of $k_{n, d}$. By replacing $X$ by the union of its Galois conjugates, we may then assume that $X$ is defined over $k_{n, d}$.

Since $\operatorname{Prep}_{f_{n, d}}(a, b)$ is finite for all $a$ and $b$, there exists an infinite sequence of tuples $\left(a_{i}, b_{i}\right)$, with $a_{i} \geq 0$ and $b_{i}>0$, so that $X$ contains a point $x_{i} \in \operatorname{Prep}_{f_{n, d}}\left(a_{i}, b_{i}\right)$ for all $i$. Since $X$ is defined over $k_{n, d}$, it follows from Propositions 3.3 and 4.6 that $\operatorname{Prep}_{f_{n, d}}\left(a_{i}, b_{i}\right) \subset X$ for all $i$. We let $X^{\prime}$ be the Zariski closure of the specialisations, over the point in Mor $_{n, d}$ corresponding to $g_{n, d}$, of the preperiodic points in $X(K)$. Since all preperiodic points lift to the generic fibre by Lemma 2.2, it follows that $X^{\prime} \subset \mathbb{P}_{k}^{n}$ has the same properties as $X$ but with respect to $\operatorname{Per}_{g_{n, d}}$.

For any $i$, the set of points in $\mathbb{P}_{\bar{k}}^{n}$ of the form $\left[\xi_{0}, \ldots, \xi_{n_{1}}, 1\right]$ with $\xi_{i} \in \operatorname{Prep}_{\phi_{0}}\left(a_{i}, b_{i}\right)$ is contained in $\operatorname{Prep}_{g_{n, d}}\left(a_{i}, b_{i}\right)$ and hence in $X^{\prime}$. As in the proof of Theorem 3.6, it then follows that $X^{\prime}=\mathbb{P}_{k}^{n}$, so $X=\mathbb{P}_{k_{n, d}}^{n}$.

Remark 4.8. Note that we do not use the full strength of the genericity hypothesis in the proofs of this section or of the previous one. It suffices to assume that the morphism under consideration corresponds to the generic point of an irreducibility subvariety of Mor $_{d, n / k}$ that contains all the families $f_{c, \epsilon}$ and is smooth at $g_{n, d}$. Since all the $f_{c, \epsilon}$ are smooth and have dimension $2 n$, there exist such subvarieties for all $n$ with dimension independent of $d$.

\section{The dynamical "Mordell-Lang” conjecture for generic endomorphisms}

Let $(X, f)$ be an algebraic dynamical system over a field $K$ of characteristic 0 ; i.e., $X$ is an algebraic variety and $f: X \rightarrow X$ is a morphism. The conjecture of Ghioca and Tucker [2009] asserts that, if $x \in X(K)$ and $Y$ a subvariety of $X$ are such that $O_{f}(x) \cap Y(K)$ is infinite, then there is a periodic subvariety $Z$ of $X$ with $Z \subset Y$ and $Z(K) \cap O_{f}(x) \neq \varnothing$. It has been proved when $f$ is étale by Bell et al. [2010] and in a few other cases. It is not known in general if $X=\mathbb{P}_{K}^{n}$ and $\operatorname{deg}(f)>1$; this was the original case investigated by Denis [1994], who proved the assertion under the assumption that $O_{f}(x) \cap Y(K)$ is large in a suitable sense.

For $(X, f)=\left(\mathbb{P}_{K}^{n}, f\right)$, with $f$ a generic endomorphism, by Theorem 3.6, there are no nontrivial $f$-periodic subvarieties contained in $\mathbb{P}_{K}^{n}$, so the conjecture in this case is equivalent to the following:

Theorem 5.1. Let $f: \mathbb{P}_{K}^{n} \rightarrow \mathbb{P}_{K}^{n}$ be a generic endomorphism of degree $d>1$ over an algebraically closed field $K$ of characteristic zero. For each $x \in \mathbb{P}^{n}(K)$, every infinite subset of $O_{f}(x)$ is Zariski-dense in $\mathbb{P}_{K}^{n}$. 
The idea of the proof is as follows. We first use specialisation to reduce to the case that $K$ is a finite extension of $k_{n, d}$. We then show using a $p$-adic argument, for a prime $p$ dividing $d$, that any $Y$ such that $O_{f}(x) \cap Y$ is infinite must contain infinitely many periodic points, or there exists a prime $q$ not diving $d$, such that $x$ and $f$ have specialisations $\bar{x}$ and $\bar{f}$ over $\mathbb{F}_{q}$ with $\bar{f}$ étale on the orbit of $\bar{x}$. Both these conditions lead to the conclusion that $Y=\mathbb{P}_{K}^{n}$, the first from Theorem 4.1 and second by using a result of Bell, Ghioca and Tucker, which we recall:

Lemma 5.2. Let $L / \mathbb{Q}_{p}$ be a finite extension, $\pi: X \rightarrow \operatorname{Spec}(R)$ a smooth scheme of finite type over the ring of integers $R$ of $L$ and $f: X \rightarrow X$ a morphism over $\operatorname{Spec}(R)$. Suppose $x \in X(R)$ is such that $f$ is étale on the orbit of $x$. If $Y \subset X$ is any closed subscheme with $Y \cap O_{f}(x)$ infinite, then $Y_{L}$ contains a positive-dimensional periodic subvariety of $X_{L}$.

Proof. If $L=\mathbb{Q}_{p}$, this is an immediate consequence of the results in [Bell et al. 2010]. For general $L$, it follows from the methods in the same work, if one replaces Theorem 3.3 therein by Theorem 7 of [Amerik 2011].

Lemma 5.3. Theorem 5.1 for arbitrary extensions $K$ of $k_{n, d}$ follows from the case of finite extensions.

Proof. Without loss of generality, we may assume that $k=\mathbb{Q}$ and $K$ is a finitely generated extension of $k_{n, d}$. Let $x \in \mathbb{P}^{n}(K)$, and let $Y$ be a subvariety of $\mathbb{P}_{K}^{n}$ such that $O_{f}(x) \cap Y(K)$ is infinite.

Since $K$ is finitely generated, there exists a smooth irreducible scheme $M$ of finite type over $\mathbb{Z}$ with function field $K$ and a dominant morphism $\pi: M \rightarrow \operatorname{Mor}_{n, d}$ inducing the inclusion $k_{n, d} \subset K$ on function fields. Let $f: M \times \mathbb{P}^{n} \rightarrow M \times \mathbb{P}^{n}$ be the pullback of the universal morphism from Mor $_{n, d} \times \mathbb{P}^{n}$, so $f$ restricted to the generic fibre of the first projection is equal to $f$. Let $\boldsymbol{x}$ and $\boldsymbol{Y}$ be the Zariski closures of $x$ and $Y$, respectively, in $M \times \mathbb{P}^{n}$. By shrinking $M$ if necessary, we may assume that $\boldsymbol{x}$ and $\boldsymbol{Y}$ are flat over $M$.

Since $p$ is a dominant finite-type morphism, there exists a point $f^{\prime}$ of $M$ (which we think of as an endomorphism of $\mathbb{P}^{n}$ using $\pi$ ) mapping to the generic point of $\operatorname{Mor}_{n, d}$ and so that the residue field $K^{\prime}$ at $f^{\prime}$ is a finite extension of $k_{n, d}$. Let $x^{\prime}$ and $Y^{\prime}$ denote the fibres of $\boldsymbol{x}$ and $\boldsymbol{Y}$, respectively, over $f^{\prime}$. If $O_{f^{\prime}}\left(x^{\prime}\right)$ is infinite, then so is $O_{f^{\prime}}\left(x^{\prime}\right) \cap Y^{\prime}$; hence, by the condition on $K^{\prime}$, it would follow that $Y^{\prime}=\mathbb{P}_{K^{\prime}}^{n}$, which (by flatness) implies $Y=\mathbb{P}_{K}^{n}$.

If $O_{f^{\prime}}\left(x^{\prime}\right)$ is finite, then $x^{\prime}$ is $f^{\prime}$-preperiodic. Since $f^{\prime}$ is generic, it follows from Corollary 3.5 that $O_{f^{\prime}}\left(x^{\prime}\right)$ does not intersect the ramification locus of $f^{\prime}$. Let $\left|O_{f^{\prime}}\left(x^{\prime}\right)\right|=n$, and let $\boldsymbol{Z}$ denote the Zariski closure of $\left\{x, f(x), \ldots, f^{n-1}(x)\right\}$ in $M \times \mathbb{P}^{n}$. Let $\boldsymbol{R} \subset M \times \mathbb{P}^{n}$ be the ramification locus of $\boldsymbol{f}$, and consider the closed subset $\boldsymbol{R} \cap \boldsymbol{Z}$ of $M \times \mathbb{P}^{n}$. By the above, the fibre of this subset over $f^{\prime}$ is empty, so 
by the properness of $\mathbb{P}^{n}$, its projection in $M$ is a proper closed subset. Replacing $M$ by the complement of this subset, we may assume that $\boldsymbol{R} \cap \boldsymbol{Z}=\varnothing$.

Now let $f^{\prime \prime}$ be any closed point (which we again think of as an endomorphism) of $M$ that lies in the closure of $f^{\prime}$. Since $M$ is of finite type over $\mathbb{Z}$, the residue field of $f^{\prime \prime}$ is a finite field $F$. Let $x^{\prime \prime}$ and $Y^{\prime \prime}$ denote the fibres of $\boldsymbol{x}$ and $\boldsymbol{Y}$, respectively, over $f^{\prime \prime}$. Since $f^{\prime \prime}$ is in the closure of $f^{\prime}, x^{\prime \prime}$ is in the closure of $x^{\prime}$; hence, $\left|O_{f^{\prime \prime}}\left(x^{\prime \prime}\right)\right| \leq n$. Since $\boldsymbol{R} \cap \boldsymbol{Z}=\varnothing$, it follows that $f^{\prime \prime}$ is unramified at all points of $O_{f^{\prime \prime}}\left(x^{\prime \prime}\right)$. Let $W(F)$ be the ring of Witt vectors of $F$. Since $M$ is smooth over $\mathbb{Z}$, by Hensel's lemma, the set of points in $M(W(F))$ that specialise to $f^{\prime \prime}$ is in bijection (after choosing local coordinates) with $W(F)^{n}$. The subset consisting of points that lie in a proper closed subscheme of $M$ is a countable union of nowhere dense (in the adic topology) subsets. It follows by Baire's theorem that there exists a point in $M(W(F))$ specialising to $f^{\prime \prime}$ and not lying in any proper closed subscheme. Letting $L$ be the quotient field of $W(K)$, it follows that the image of the induced map from $\operatorname{Spec}(L)$ to $M$ must be the generic point. We thus get an inclusion of $K$ into $L$, and we may apply Lemma 5.2 with $R=W(K)$ and $X=\operatorname{Spec}(W(F)) \times \mathbb{P}^{n}$ to the base change of $\boldsymbol{f}, \boldsymbol{x}$ and $\boldsymbol{Y}$ via the morphism $\operatorname{Spec}(W(F)) \rightarrow M$ to conclude using Theorem 3.6(1) that $Y=\mathbb{P}_{K}^{n}$.

Lemma 5.4. Let $p$ be a prime and $g_{n, d, p}$ denote the endomorphism of $\mathbb{P}_{\mathbb{F}_{p}}^{n}$ given by raising each coordinate to its $d$-th power. Let $X \subset \mathbb{P}_{\overline{\mathbb{F}}_{p}}^{n}$ be a positive-dimensional subvariety. Then the set $\bigcup_{r \geq 0} g_{n, d, p}^{r}\left(X\left(\overline{\mathbb{F}}_{p}\right)\right)$ contains periodic points of infinitely
many distinct periods.

Proof. Since $g_{n, d, p}$ preserves the standard decomposition of $\mathbb{P}^{n}$ as a disjoint union of affine spaces, by projecting to a suitable coordinate, we reduce to the statement for $n=1$, in which case the statement is obvious.

Remark 5.5. We expect that the lemma holds with $g_{n, d, p}$ replaced by an arbitrary quasipolarised morphism - or even more generally with some extra conditions on $X$ - defined over a finite field, but this seems much harder to prove. However, for endomorphisms of abelian varieties the corresponding statement can indeed be proved.

Lemma 5.6. Let $d>1$ be an integer and $p$ a prime such that $p \mid d$. Let $\phi$ be the morphism $\mathbb{A}^{2} \rightarrow \mathbb{A}^{2}$ given by $\phi(x, c)=\left(x^{d}+c, c\right)$ over the field $\overline{\mathbb{F}}_{p}$.

(a) For $c \in \overline{\mathbb{F}}_{p}$, the monodromy action on the set of fixed points of $\phi_{c}$ is transitive.

(b) Let $X \subset \mathbb{A}^{2}$ be an irreducible subvariety of dimension 1, mapping dominantly to $\mathbb{A}^{1}$ via the second projection $p_{2}$. Assume that the intersection of $X$ with the generic fibre of $p_{2}$ is not a preperiodic point of $\phi$. Then the $\phi$-periods of the points in $X\left(\overline{\mathbb{F}}_{p}\right)$ (which are all preperiodic) are unbounded. 
Proof. We will use elementary intersection theory on $\mathbb{P}^{1} \times Y$ with $Y$ a smooth projective curve.

Let $X_{n}=\phi^{n}(X)$ for $n \geq 0$. By replacing $X$ by $\phi^{r}(X)$ for some $r \geq 0$, we assume that the map $p_{2}: X_{n} \rightarrow \mathbb{P}^{1}$ has degree $e$ for all $n$.

For any integer $b>0$, let $P_{b}$ be the locus of points in $\mathbb{A}^{2}$ that are $\phi$-periodic of period $b$. Since $p \mid d, \phi$ is inseparable on the fibres of $p_{2}$, so the graph of $\left.\phi^{b}\right|_{\mathbb{P}^{1} \times\{c\}}$ intersects the diagonal in $\mathbb{A}^{2}$ transversely for all $c \in \mathbb{A}^{1}$ and all $b$. It follows that $P_{b}$ is a finite étale cover of $\mathbb{A}^{1}$ via $p_{2}$ and $P_{b} \cap P_{b^{\prime}}=\varnothing$ for $b \neq b^{\prime}$.

Let $\bar{P}_{b}$ be the closure of $P_{b}$ in $\mathbb{P}^{1} \times \mathbb{P}^{1} \supset \mathbb{A}^{1} \times \mathbb{A}^{1}=\mathbb{A}^{2}$, and let $\bar{X}_{n}$ be the closure of $X_{n}$ in $\mathbb{P}^{1} \times \mathbb{P}^{1}$. The curve $P_{b}$ is a subcurve of the curve $Q_{b}$ in $\mathbb{A}^{2}$ with equation

$$
\left(\ldots\left(\left(x^{d}+c\right)^{d}+c\right)^{d} \ldots\right)^{d}+c-x=0,
$$

where we have $b$ pairs of brackets. Replacing $c$ by $1 / c^{\prime}$ in the above equation and multiplying through by $c^{\prime} d^{(b-1)}$, we get the equation

$$
\left(\ldots\left(\left(c^{\prime} x^{d}+1\right)^{d}+c^{\prime} d^{b-1}-d^{b-2}\right)^{d} \ldots\right)^{d}+c^{\prime} d^{b-1}-1-c^{\prime d^{b-1}} x=0 .
$$

It follows that the only point on all the $\bar{P}_{b}$ intersecting the fibre over $c=\infty$ is the point at infinity on this fibre and the support of $\bar{P}_{b} \cap \bar{P}_{b^{\prime}}$ is equal to this point if $b \neq b^{\prime}$. When $b=1$, the equation is

$$
c^{\prime} x^{d}+1-c^{\prime} x=0 .
$$

One then sees that $\bar{P}_{1}$ is irreducible since the equation shows that it is smooth at the point $(\infty, \infty)$ and the closure in $\mathbb{P}^{1} \times \mathbb{P}^{1}$ of any irreducible component of $P_{1}$ must contain this point; this proves (a).

If the $\phi$-periods of all points in $X\left(\mathbb{\mathbb { F }}_{p}\right)$ are bounded, then there must exist some $b>0$ so that $\left|\bar{X}_{n} \cap \bar{P}_{b}\right| \rightarrow \infty$ as $n \rightarrow \infty$ and, by assumption, $\bar{X}_{n} \nsubseteq \bar{P}_{b}$ for all $b$. Writing $\left[\bar{X}_{n}\right]=e\left[\{0\} \times \mathbb{P}^{1}\right]+a_{n}\left[\mathbb{P}^{1} \times\{0\}\right]$ in $\mathrm{NS}\left(\mathbb{P}^{1} \times \mathbb{P}^{1}\right)$, it follows that $a_{n} \rightarrow \infty$ as $n \rightarrow \infty$.

Let $\bar{X}^{\prime}$ be the normalisation of $\bar{X}$, and denote by $\eta: \bar{X}^{\prime} \rightarrow \mathbb{P}^{1}$ the composition of the normalisation map and the projection $p_{2}$. Let $x_{1}, x_{2}, \ldots, x_{r}$ be the points in $\bar{X}$ mapping to the point $(\infty, \infty)$ in $\mathbb{P}^{1} \times \mathbb{P}^{1}$. If $r=0$, it follows that $X \cap P_{b} \neq \varnothing$ for all $b$, so we may assume that $r>0$. Let $b_{1}, b_{2}, \ldots, b_{r+1}$ be any distinct integers, and let $\gamma: Y \rightarrow \bar{X}^{\prime}$ be a Galois cover such that there are components $S_{i}$ of $(\mathrm{id} \times \eta \gamma)^{-1}\left(\bar{P}_{b_{i}}\right)$ in $\mathbb{P}^{1} \times Y$ that map isomorphically to $Y$ via the second projection. Let $Z$ be the section of this projection induced by the tautological section of $\mathbb{P}^{1} \times \bar{X}^{\prime}$, and let $Z_{n}=\psi^{n}(Z)$, where $\psi$ is the map of $\mathbb{P}^{1} \times Y$ induced by $\phi$. We may write $\left[S_{i}\right]=[* \times Y]+s_{i}\left[\mathbb{P}^{1} \times *\right]$ and $\left[Z_{n}\right]=[* \times Y]+a_{n}\left[\mathbb{P}^{1} \times *\right]$ in $\mathrm{NS}\left(\mathbb{P}^{1} \times Y\right)$ where $s_{i} \geq 0$ and $*$ denotes any point. It follows that the intersection number $S_{i} \cdot Z_{n}=s_{i}+a_{n} \rightarrow \infty$ as $n \rightarrow \infty$ for all $i=1,2, \ldots, r$. 
Now consider the local intersection multiplicity $I_{y}\left(S_{i}, Z_{n}\right)$ of $S_{i}$ and $Z_{n}$ at a point $\infty \times y$, where $y \in Y$ is such that $\gamma(y)=x_{j}$ for some $j$. If this is bounded for all such $y$ and all $n$, since $S_{i} \cdot Z_{n} \rightarrow \infty$, it would follow that for large $n, S_{i}$ and $Z_{n}$ must intersect in a point $\left(z, y^{\prime}\right)$ such that $\eta \gamma\left(y^{\prime}\right) \in \mathbb{A}^{1} \subset \mathbb{P}^{1}$, which implies that $X_{n} \cap P_{b_{i}} \neq \varnothing$.

Suppose this is not the case, so $I_{y}\left(S_{i}, Z_{n_{l}}\right) \rightarrow \infty$ for some infinite sequence $n_{l} \rightarrow \infty$. Since the $S_{i}$ are all distinct smooth curves and there are only finitely many of them, it follows that $I_{y}\left(T, Z_{n_{l}}\right)$ must remain bounded as $n_{l} \rightarrow \infty$, where $T$ runs over all $S_{i^{\prime}}$ for $i^{\prime} \neq i$ and all of their Galois conjugates. Up to Galois conjugation, there are only $r$ points $y$ as above, so it follows that we must have that, for all large $n_{l}$, there exists $i_{n_{l}} \in\{1,2, \ldots, r+1\}$ so that $I_{y}\left(S_{i_{n_{l}}}, Z_{n_{l}}\right)$ is bounded by an integer independent of $n_{l}$ for all $y$ as above. It follows that we must have $X_{n_{l}} \cap P_{b_{i_{l}}} \neq \varnothing$.

By choosing infinitely many disjoint sets of $r+1$ distinct integers $\left\{b_{1}, b_{2}, \ldots\right.$, $\left.b_{r+1}\right\}$ as above, we see that $X_{n_{b}} \cap P_{b} \neq \varnothing$ for infinitely many distinct integers $b$ (and $n_{b}$ depending on $b$ ). Since all the $P_{b}$ are disjoint, it follows that $X$ contains preperiodic points of infinitely many distinct periods.

Remark 5.7. We also expect this lemma to hold in much greater generality, e.g., for any 1-parameter family of maps defined over $\overline{\mathbb{F}}_{p}$.

The following lemma is the key to our construction of a periodic point in $Y$ under the assumption that $O(x) \cap Y(K)$ is infinite:

Lemma 5.8. Let $L / \mathbb{Q}_{p}$ be a finite extension, $\pi: X \rightarrow \operatorname{Spec}(R)$ a smooth projective scheme over the ring of integers $R$ of $L$ and $f: X \rightarrow X$ a quasipolarised morphism over $\operatorname{Spec}(R)$. Assume that the differential of $f, d f$, is 0 on the special fibre of $X$. For any $x \in X(L)=X(R)$, let $b$ be the period of the reduction $\bar{x}$ of $x$ in the special fibre of $X$. Then for any integer $a \geq 0$, the sequence of points $f^{a+b n}(x)$ converges to a periodic point of $X(L)$ of period $b$.

Proof. Replacing $f$ by $f^{n}$ and $x$ by $f^{a^{\prime}}(x)$, for any integer $a^{\prime}$ greater than the preperiod of $\bar{x}$, we may assume that $\bar{x}$ is a fixed point of $f$, and we then need to prove that $f^{n}(x)$ converges to a fixed point.

Since $f$ is quasipolarised, by Lemma 2.2(2), $\bar{x}$ lifts to a fixed point $y$ of $f$ defined over a finite extension of $L$; by replacing $L$ by this extension, we may assume that $y \in X(L)$.

Let $A$ be the completion of the local ring of $\bar{x}$ on $X$. Since $\pi$ is smooth, $A \cong R \llbracket z_{1}, z_{2}, \ldots, z_{n} \rrbracket$, where $n+1=\operatorname{dim}(X)$. Using any such isomorphism, the set of points of $X(L)$ that specialise to $\bar{x}$ is identified with the set $\left(m_{R}\right)^{n}$, where $m_{R}$ is the maximal ideal of $R$. We fix such an isomorphism, which we also assume identifies $y$ with $(0, \ldots, 0) \in\left(m_{R}\right)^{n}$.

Since $\bar{x}$ is a fixed point of $f, f$ induces an endomorphism of $A$ that, with respect to the chosen isomorphism, is given by an $n$-tuple of elements $\left(f_{1}, f_{2}, \ldots, f_{n}\right)$ in 
the maximal ideal of the local ring $R \llbracket z_{1}, z_{2}, \ldots, z_{n} \rrbracket$. Moreover, since $f$ fixes $y$, it follows that the constant term of each $f_{i}$ is 0 . Since $d f$ is assumed to be zero on the special fibre of $X$, it follows that the coefficients of the linear term of each $f_{i}$ lies in $m_{R}$. For any $\lambda=\left(\lambda_{1}, \lambda_{2}, \ldots, \lambda_{n}\right) \in\left(m_{R}\right)^{n}$, let $|\lambda|=\max _{i}\left\{\left|\lambda_{i}\right|\right\}$. The conditions on the $f_{i}$ imply that, for any such $\lambda,|f(\lambda)|<|\lambda|$ if $\lambda \neq(0, \ldots, 0)$. Since $R$ is a discrete valuation ring, it follows that for any such $\lambda$ we have that $f^{n}(\lambda) \rightarrow(0,0, \ldots, 0)$ as $n \rightarrow \infty$; hence, $f^{n}(x) \rightarrow y$ as $n \rightarrow \infty$.

Proof of Theorem 5.1. By Lemma 5.3, we may assume that $K$ is a finite extension of $k_{n, d}$. Furthermore, we may assume without loss of generality that our base field $k=\mathbb{Q}$.

Let $x \in \mathbb{P}^{n}(K)$, and assume that $Y$ is a subvariety defined over $K$ such that $I=O(x) \cap Y(K)$ is infinite. Let $X$ be the Zariski closure of the image of $x$ in Mor $_{n, d} \times \mathbb{P}_{\mathbb{Z}}^{n}$, and let $\chi$ denote the map $X \rightarrow$ Mor $_{n, d}$ induced by projection to the first factor.

Let $p$ be a prime dividing $d$. Since $\operatorname{Mor}_{n, d}$ is smooth over $\operatorname{Spec}(\mathbb{Z})$, there is a map $g: \operatorname{Spec}\left(\mathbb{Z}_{p}\right) \rightarrow \operatorname{Mor}_{n, d}$ such that the generic point of $\operatorname{Spec}\left(\mathbb{Z}_{p}\right)$ maps to the generic point of Mor $_{n, d}$ and the closed point maps to the point corresponding to $g_{d, n, p}$, the $d$-power map over $\mathbb{F}_{p}$. Since $p \mid d$, the differential of the endomorphism of $\mathbb{P}_{\mathbb{Z}_{p}}^{n}$ corresponding to $g$, which we also denote by $g$, is zero on the special fibre. Suppose the fibre $X_{g_{d, n, p}}$ of $\chi$ over $g_{d, n, p}$ is infinite. By Lemma 5.4, the set $\bigcup_{r \geq 0} g_{d, n, p}^{r}\left(X_{g_{d, n, p}}\left(\overline{\mathbb{F}}_{p}\right)\right)$ contains infinitely many periodic points. By applying Lemma 5.8, we can lift all these periodic points to periodic points of $f_{n, d}$ contained in $Y$. It then follows from Theorem 4.1 that $Y=\mathbb{P}_{K}^{n}$. Thus, we may assume from now on that $\chi$ is finite over an open neighbourhood of $g_{n, d, p}$.

By replacing $x$ by $f^{r}(x)$ for some large $r$, we may assume that $X_{g_{d, n, p}}$ contains a periodic point $x^{\prime}=\left[x_{0}^{\prime}, x_{1}^{\prime}, \ldots, x_{n}^{\prime}\right]$ with $x_{i}^{\prime} \in \overline{\mathbb{F}}_{p}$. Since $\chi$ is finite in a neighbourhood of $g_{n, d, p}$, Mor $_{d, n}$ is smooth, hence normal, and $X$ is irreducible, it follows from the going-down theorem that if none of the $x_{i}^{\prime}=0$ then the fibre $X_{g_{n, d}}$ of $\chi$ over $g_{n, d}$ contains a point $\tilde{x}^{\prime}$ lifting $x^{\prime}$. By specialisation, it follows that for all large primes $q$ the fibre of $\chi$ over $g_{n, d, q}$ contains a point all of whose coordinates are nonzero or, equivalently, not contained in the ramification locus of $g_{n, d, q}$. Since this locus is invariant under $g_{n, d, q}$, we may apply Lemma 5.2 to conclude the existence of a positivedimensional periodic subvariety of $Y$, which, by Theorem 3.6, implies $Y=\mathbb{P}_{K}^{n}$.

We now use Lemma 5.6 to show that such an $x^{\prime}$ must exist, at least after replacing $x$ by a Galois conjugate, or $Y$ must contain infinitely many periodic points, both cases leading to the conclusion that $Y=\mathbb{P}_{K}^{n}$. Let $x^{\prime}$ be as above, and suppose that $x_{0}^{\prime}=0$. Some other coordinate must be nonzero, so by symmetry, we may assume that $x_{n}^{\prime} \neq 0$, and then by multiplying through by a scalar, we may assume $x_{n}^{\prime}=1$. 
Consider the family of endomorphisms $\psi_{c}$ of $\mathbb{P}_{\mathbb{F}_{p}}^{n}$ parametrised by $\mathbb{A}^{1}$ given by

$$
\psi_{c}\left(\left[x_{0}, x_{1}, \ldots, x_{n}\right]\right)=\left[x_{0}^{d}+c x_{n}^{d}, x_{1}^{d}, \ldots, x_{n}^{d}\right],
$$

so $\psi_{0}=g_{n, d, p}$. Note that, on the affine space given by the locus with $x_{n} \neq 0, \psi_{c}$ is given in affine coordinates by $\left(z_{0}, z_{1}, \ldots, z_{n-1}\right) \mapsto\left(z_{0}^{d}+c, z_{1}^{d}, \ldots, z_{n-1}^{d}\right)$.

Let $S \subset$ Mor $_{n, d}$ be the subscheme corresponding to the family $\psi_{c}$. By the goingdown theorem, there is an irreducible component $T$ of $\chi^{-1}(S)$ mapping onto $S$ and containing the point $\left(g_{n, d, p}, x^{\prime}\right)$. Let $T^{\prime}$ be the image of $T$ in $\mathbb{P}_{\mathbb{F}_{p}}^{n}$ under the projection of $X$ to $\mathbb{P}^{n}$. Since $x_{n}^{\prime}=1, T^{\prime}$ is not contained in the locus given by $x_{n}=0$, so by projecting to the first $n$ coordinates, we get a rational map $\rho$ from $T$ to $\mathbb{A}^{n}$.

Suppose the composition of $\rho$ with the $i$-th projection is nonconstant for some $i$, $0<i \leq n-1$. Since the action of $\psi_{c}$ on the $i$-th coordinate doesn't depend on $c$, it follows that $T\left(\overline{\mathbb{F}}_{p}\right)$ must contain preperiodic points of arbitrarily large period. By Lemma 5.8 as before, we obtain infinitely many periodic points in $Y$, forcing $Y=\mathbb{P}_{K}^{n}$.

So suppose $\rho$ composed with all the $i$-th projections are constant for $i>0$, and let $\sigma: T \rightarrow \mathbb{A}^{1} \times S$ be given by $\left(\pi_{0} \rho, \chi\right)$. By applying Lemma 5.6(b), it follows that if the image of $T$ is not contained inside a preperiodic curve for the map $\phi$ (using the identification of $S$ with $\mathbb{A}^{1}$ ) there must be $\phi$ preperiodic points in the image with unbounded period. By the construction of $\psi_{d}$, it follows that there are preperiodic points on $T$ of unbounded period. As before, this implies that $Y=\mathbb{P}^{n}$.

The last case we need to consider is when the image of $T$ lies in a preperiodic curve. By replacing $x$ by an element in its orbit if necessary, we may assume that this image lies in the periodic locus. Now 0 is a fixed point of the map $z \mapsto z^{d}$, and the point $(0,0)$ is contained in the image of $T$ by construction. By Lemma 5.6(a), it follows that the point $(0,1)$ is also in the image of $T$. We conclude that $X_{g_{d, n, p}}$ contains the periodic point $x^{\prime \prime}=\left[1, x_{1}^{\prime}, \ldots, x_{n-1}^{\prime}, 1\right]$. By replacing $x^{\prime}$ with $x^{\prime \prime}$ and repeating the above argument if necessary, we conclude that $Y$ contains infinitely many periodic points, in which case it must be $\mathbb{P}_{k}^{n}$, or $X_{g_{d, n, p}}$ contains a periodic point $x^{\prime}=\left[x_{0}^{\prime}, x_{1}^{\prime}, \ldots, x_{n}^{\prime}\right]$ with $x_{i}^{\prime} \neq 0$ for all $i$. As we have already seen, this also implies that $Y=\mathbb{P}_{K}^{n}$, concluding the proof.

Remark 5.9. Note that a statement similar to Remark 4.8 holds: it suffices to consider generic points of irreducible subschemes of Mor $_{d, n}$ that contain all the families $f_{i}$ and are smooth at the point $g_{n, d, p}$ for some prime $p$ dividing $d$.

\section{Acknowledgements}

I thank Ekaterina Amerik, Amitava Bhattacharya, Bjorn Poonen, Joseph Silverman and Thomas Tucker for useful conversations and comments. I also thank the referee for his/her suggestions for improvements in the exposition. 


\section{References}

[Amerik 2011] E. Amerik, "Existence of non-preperiodic algebraic points for a rational self-map of infinite order", Math. Res. Lett. 18:2 (2011), 251-256. MR 2012e:37182 Zbl 1241.14011

[Bell et al. 2010] J. P. Bell, D. Ghioca, and T. J. Tucker, "The dynamical Mordell-Lang problem for étale maps", Amer. J. Math. 132:6 (2010), 1655-1675. MR 2012a:37202 Zbl 1230.37112

[Bousch 1992] T. Bousch, Sur quelques problemes de dynamique holomorphe, $\mathrm{Ph} . \mathrm{D}$. thesis, Université de Paris-Sud, 1992.

[Denis 1994] L. Denis, “Géométrie et suites récurrentes”, Bull. Soc. Math. France 122:1 (1994), 13-27. MR 95b:11065 Zbl 0795.14008

[Douady and Hubbard 1984] A. Douady and J. H. Hubbard, Étude dynamique des polynômes complexes, I, Publications Mathématiques d'Orsay 84, Université de Paris-Sud, 1984. MR 87f:58072a Zbl 0552.30018

[Douady and Hubbard 1985] A. Douady and J. H. Hubbard, Étude dynamique des polynômes complexes, II, Publications Mathématiques d'Orsay 85, Université de Paris-Sud, 1985. MR 87f:58072b Zbl 0571.30026

[Eberlein 1999] D. Eberlein, Rational parameter rays of multibrot sets, Master's thesis, Technische Universität München, 1999.

[Fakhruddin 2003] N. Fakhruddin, "Questions on self maps of algebraic varieties", J. Ramanujan Math. Soc. 18:2 (2003), 109-122. MR 2004f:14038 Zbl 1053.14025

[Ghioca and Tucker 2009] D. Ghioca and T. J. Tucker, "Periodic points, linearizing maps, and the dynamical Mordell-Lang problem”, J. Number Theory 129:6 (2009), 1392-1403. MR 2010i:37219 Zbl 1186.14047

[Ghioca et al. 2011] D. Ghioca, T. J. Tucker, and S. Zhang, "Towards a dynamical Manin-Mumford conjecture", Int. Math. Res. Not. 2011:22 (2011), 5109-5122. MR 2012i:37142 Zbl 1267.37110

[Lau and Schleicher 1994] E. Lau and D. Schleicher, "Internal addresses in the Mandelbrot set and irreducibility of polynomials", preprint, Stony Brook University, 1994. arXiv math/9411238v1

[Morton 1998] P. Morton, "Galois groups of periodic points", J. Algebra 201:2 (1998), 401-428. MR 99a:12005 Zbl 0913.12005

[Tao and Vu 2006] T. Tao and V. Vu, Additive combinatorics, Cambridge Studies in Advanced Mathematics 105, Cambridge University Press, 2006. MR 2008a:11002 Zbl 1127.11002

Communicated by Joseph Silverman

Received 2012-11-30 Revised 2013-05-25 Accepted 2013-07-04

naf@math.tifr.res.in School of Mathematics, Tata Institute of Fundamental Research, Homi Bhabha Road, Colaba, Mumbai 400005, India 


\section{Algebra \& Number Theory}

msp.org/ant

\section{EDITORS}

MANAGING EDITOR

Bjorn Poonen

Massachusetts Institute of Technology

Cambridge, USA

\author{
EDITORIAL BOARD CHAIR \\ David Eisenbud \\ University of California \\ Berkeley, USA
}

\section{BOARD OF EDITORS}

Georgia Benkart

Dave Benson

Richard E. Borcherds

John H. Coates

J-L. Colliot-Thélène

Brian D. Conrad

Hélène Esnault

Hubert Flenner

Edward Frenkel

Andrew Granville

Joseph Gubeladze

Roger Heath-Brown

Ehud Hrushovski

Craig Huneke

Mikhail Kapranov

Yujiro Kawamata

János Kollár

Yuri Manin

Barry Mazur

Philippe Michel
University of Wisconsin, Madison, USA

University of Aberdeen, Scotland

University of California, Berkeley, USA

University of Cambridge, UK

CNRS, Université Paris-Sud, France

University of Michigan, USA

Freie Universität Berlin, Germany

Ruhr-Universität, Germany

University of California, Berkeley, USA

Université de Montréal, Canada

San Francisco State University, USA

Oxford University, UK

Hebrew University, Israel

University of Virginia, USA

Yale University, USA

University of Tokyo, Japan

Princeton University, USA

Northwestern University, USA

Harvard University, USA

École Polytechnique Fédérale de Lausanne
Susan Montgomery

Shigefumi Mori

Raman Parimala

Jonathan Pila

Victor Reiner

Karl Rubin

Peter Sarnak

Joseph H. Silverman

Michael Singer

Vasudevan Srinivas

J. Toby Stafford

Bernd Sturmfels

Richard Taylor

Ravi Vakil

Michel van den Bergh

Marie-France Vignéras

Kei-Ichi Watanabe

Efim Zelmanov

Shou-Wu Zhang
University of Southern California, USA

RIMS, Kyoto University, Japan

Emory University, USA

University of Oxford, UK

University of Minnesota, USA

University of California, Irvine, USA

Princeton University, USA

Brown University, USA

North Carolina State University, USA

Tata Inst. of Fund. Research, India

University of Michigan, USA

University of California, Berkeley, USA

Harvard University, USA

Stanford University, USA

Hasselt University, Belgium

Université Paris VII, France

Nihon University, Japan

University of California, San Diego, USA

Princeton University, USA

PRODUCTION

production@msp.org

Silvio Levy, Scientific Editor

See inside back cover or msp.org/ant for submission instructions.

The subscription price for 2014 is US $\$ 225 /$ year for the electronic version, and $\$ 400 /$ year $(+\$ 55$, if shipping outside the US) for print and electronic. Subscriptions, requests for back issues and changes of subscribers address should be sent to MSP.

Algebra \& Number Theory (ISSN 1944-7833 electronic, 1937-0652 printed) at Mathematical Sciences Publishers, 798 Evans Hall \#3840, c/o University of California, Berkeley, CA 94720-3840 is published continuously online. Periodical rate postage paid at Berkeley, CA 94704, and additional mailing offices.

ANT peer review and production are managed by EditFLOW ${ }^{\circledR}$ from Mathematical Sciences Publishers.

\section{PUBLISHED BY}

- mathematical sciences publishers

nonprofit scientific publishing

http://msp.org/

(C) 2014 Mathematical Sciences Publishers 


\section{Algebra \& Number Theory}

Volume $8 \quad$ No. $3 \quad 2014$

Derived invariants of irregular varieties and Hochschild homology

LUIGI LOMBARDI

Sato-Tate distributions of twists of $y^{2}=x^{5}-x$ and $y^{2}=x^{6}+1$

FRANCESC Fité and ANDREW V. SUTHERLAND

The algebraic dynamics of generic endomorphisms of $\mathbb{P}^{n}$

NAJMUDDIN FAKHRUDDIN

The tame-wild principle for discriminant relations for number fields

JOHN W. JONES and DAVID P. ROBERTS

Linear forms in logarithms and integral points on higher-dimensional varieties

AARON LEVIN

Lefschetz theorem for abelian fundamental group with modulus

689

MORITZ KERZ and ShuJi SAITO

Localization of spherical varieties

703

FRIEDRICH KNOP

Lefschetz operator and local Langlands modulo $\ell$ : the limit case

JEAN-FRANÇOIS DAT

Splitting tower and degree of tt-rings

PAUL BALMER 